\title{
L-space surgery and twisting operation
}

\author{
KIMIHIKO MOTEGI
}

\begin{abstract}
A knot in the 3-sphere is called an L-space knot if it admits a nontrivial Dehn surgery yielding an L-space, ie a rational homology 3-sphere with the smallest possible Heegaard Floer homology. Given a knot $K$, take an unknotted circle $c$ and twist $K n$ times along $c$ to obtain a twist family $\left\{K_{n}\right\}$. We give a sufficient condition for $\left\{K_{n}\right\}$ to contain infinitely many L-space knots. As an application we show that for each torus knot and each hyperbolic Berge knot $K$, we can take $c$ so that the twist family $\left\{K_{n}\right\}$ contains infinitely many hyperbolic L-space knots. We also demonstrate that there is a twist family of hyperbolic L-space knots each member of which has tunnel number greater than one.
\end{abstract}

57M25, 57M27; 57N10

\section{Introduction}

Heegaard Floer theory (with $\mathbb{Z} / 2 \mathbb{Z}$ coefficients) associates a group $\widehat{\mathrm{HF}}(M, \boldsymbol{t})$ to a closed, orientable $\operatorname{spin}^{c} 3$-manifold $(M, t)$. The direct sum of $\widehat{\mathrm{HF}}(M, t)$ for all $\operatorname{spin}^{c}$ structures is denoted by $\widehat{\mathrm{HF}}(M)$. A rational homology 3 -sphere $M$ is called an $L$-space if $\widehat{\mathrm{HF}}(M, \boldsymbol{t})$ is isomorphic to $\mathbb{Z} / 2 \mathbb{Z}$ for all $\operatorname{spin}^{c}$ structures $\boldsymbol{t} \in \operatorname{Spin}^{c}(M)$. Equivalently, the dimension $\operatorname{dim}_{\mathbb{Z} / 2 \mathbb{Z}} \widehat{\mathrm{HF}}(M)$ is equal to the order $\left|H_{1}(M ; \mathbb{Z})\right|$. A knot $K$ in the 3-sphere $S^{3}$ is called an $L$-space knot if the result $K(r)$ of $r$-surgery on $K$ is an L-space for some nonzero integer $r$, and the pair $(K, r)$ is called an $L$-space surgery. The class of L-spaces includes lens spaces (except $S^{2} \times S^{1}$ ), and more generally, 3-manifolds with elliptic geometry; see Ozsváth and Szabó [47, Proposition 2.3]. Since the trivial knot, nontrivial torus knots and Berge knots [5] admit nontrivial surgeries yielding lens spaces, these are fundamental examples of L-space knots. For the mirror image $K^{*}$ of $K, K^{*}(-r)$ is homeomorphic to $K(r)$ with the opposite orientation. So if $K(r)$ is an L-space, then $K^{*}(-r)$ is also an L-space [47, page 1288]. Hence if $K$ is an L-space knot, then so is $K^{*}$.

Let $K$ be a nontrivial L-space knot with a positive L-space surgery. Then Ozsváth and Szabó prove in [48, Proposition 9.6] (see also Hedden [25, Lemma 2.13]) that $r$-surgery on $K$ results in an L-space if and only if $r \geq 2 g(K)-1$, where $g(K)$ denotes the genus of $K$. This result, together with Thurston's hyperbolic Dehn surgery 
theorem (see [51; 52] and also Benedetti and Petronio [4], Petronio and Porti [49], and Boileau and Porti [7]), shows that each hyperbolic L-space knot, say a hyperbolic Berge knot, produces infinitely many hyperbolic L-spaces by Dehn surgery.

On the other hand, there are some strong constraints for L-space knots:

- The nonzero coefficients of the Alexander polynomial of an L-space knot are \pm 1 and alternate in sign [47, Corollary 1.3].

- An L-space knot is fibered; see Ni [43, Corollary 1.2] and [44], and also Ghiggini [19] and Juhász [29].

- An L-space knot is prime; see Krcatovich [31, Theorem 1.2].

Note that these conditions are not sufficient. For instance, $10_{132}$ satisfies the above conditions, but it is not an L-space knot; see [47].

As shown in Hedden [25] and Hom, Lidman and Vafaee [26], some satellite operations keep the property of being L-space knots. In the present article, we consider whether some suitably chosen twistings also keep the property of being L-space knots. Given a knot $K$, take an unknotted circle $c$ which bounds a disk intersecting $K$ at least twice. Then performing an $n$-twist, ie $(-1 / n)$-surgery along $c$, we obtain another knot $K_{n}$. Then our question is formulated as:

Question 1.1 Which knots $K$ admit an unknotted circle $c$ such that an $n$-twist along $c$ converts $K$ into an L-space knot $K_{n}$ for infinitely many integers $n$ ? Furthermore, if $K$ has such a circle $c$, which circles enjoy the desired property?

Example 1.2 Let $K$ be a pretzel knot $P(-2,3,7)$, and take an unknotted circle $c$ as in Figure 1. Then following Ozsváth and Szabó [47], $K_{n}$ is an L-space knot if $n \geq-3$ and thus the twist family $\left\{K_{n}\right\}$ contains infinity many L-space knots. Note that this family, together with a twist family $\left\{T_{2 n+1,2}\right\}$, comprise all Montesinos L-space knots; see Lidman and Moore [33] and Baker and Moore [3].

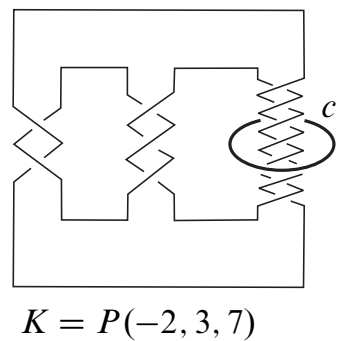

Figure 1: A knot $K_{n}$ obtained by $n$-twist along $c$ is an L-space knot if $n \geq-3$. 
In this example, it turns out that $c$ becomes a Seifert fiber in the lens space $K(19)$ (see Figure 10). We employed such a circle for relating Seifert fibered surgeries in Deruelle, Miyazaki and Motegi [13]. A pair $(K, m)$ of a knot $K$ in $S^{3}$ and an integer $m$ is a Seifert surgery if $K(m)$ has a Seifert fibration; we allow the fibration to be degenerate, ie it contains an exceptional fiber of index 0 as a degenerate fiber. See [13, Section 2.1] for details. The definition below enables us to say that $c$ is a seiferter for the Seifert (lens space) surgery $(K, 19)$.

Definition 1.3 [13] Let $(K, m)$ be a Seifert surgery. A knot $c$ in $S^{3}-N(K)$ is called a seiferter for $(K, m)$ if $c$ satisfies the following:

- $c$ is a trivial knot in $S^{3}$.

- $c$ becomes a fiber in a Seifert fibration of $K(m)$.

As remarked in [13, Convention 2.15], if $c$ bounds a disk in $S^{3}-K$, then we do not regard $c$ as a seiferter. Thus for any seiferter $c$ for $(K, m), S^{3}-$ int $N(K \cup c)$ is irreducible.

Let $(K, m)$ be a Seifert surgery with a seiferter $c$. There are two cases: either $c$ becomes a fiber in a nondegenerate Seifert fibration of $K(m)$ or $c$ becomes a fiber in a degenerate Seifert fibration of $K(m)$. In the former case, for homological reasons, the base surface is the 2 -sphere $S^{2}$ or the projective plane $\mathbb{R} P^{2}$. Suppose that $c$ is a fiber in a nondegenerate Seifert fibration of $K(m)$ over the 2 -sphere $S^{2}$. Then in the following we assume that $K(m)$ contains at most three exceptional fibers, and if there are three exceptional fibers, then $c$ is an exceptional fiber. We call such a seiferter a seiferter for a small Seifert fibered surgery $(K, m)$. To be precise, the images of $K$ and $m$ after an $n$-twist along $c$ should be denoted by $K_{c, n}$ and $m_{c, n}$, but for simplicity, we abbreviate them to $K_{n}$ and $m_{n}$ respectively as long as there is no confusion.

Theorem 1.4 Let $c$ be a seiferter for a small Seifert fibered surgery $(K, m)$. Then $\left(K_{n}, m_{n}\right)$ is an L-space surgery for an infinite interval of integers $n$ if and only if the result of $(m, 0)$-surgery on $K \cup c$ is an $L$-space.

In remaining cases, it turns out that every seiferter enjoys the desired property in Question 1.1.

Theorem 1.5 Let $c$ be a seiferter for $(K, m)$ that becomes a fiber in a Seifert fibration of $K(m)$ over $\mathbb{R} P^{2}$. Then $\left(K_{n}, m_{n}\right)$ is an L-space surgery for all but at most one integer $n_{0}$ with $\left(K_{n_{0}}, m_{n_{0}}\right)=(O, 0)$. Hence $K_{n}$ is an L-space knot for all integers $n$. 
Let us turn to the case where $c$ is a (degenerate or nondegenerate) fiber in a degenerate Seifert fibration of $K(m)$. Recall from [13, Proposition 2.8] that if $K(m)$ has a degenerate Seifert fibration, then it is a lens space or a connected sum of two lens spaces such that neither summand is $S^{3}$ or $S^{2} \times S^{1}$. The latter 3-manifold will be simply referred to as a connected sum of two lens spaces, which is an L-space; see Szabó [50, page 221] and also Ozsváth and Szabó [46, Proposition 6.1].

Theorem 1.6 Let $c$ be a seiferter for $(K, m)$ which becomes a (degenerate or nondegenerate) fiber in a degenerate Seifert fibration of $K(\mathrm{~m})$.

(1) If $K(m)$ is a lens space, then $\left(K_{n}, m_{n}\right)$ is an L-space surgery; hence $K_{n}$ is an $L$-space knot for all but at most one integer $n$.

(2) If $K(m)$ is a connected sum of two lens spaces, then $\left(K_{n}, m_{n}\right)$ is an L-space surgery, hence $K_{n}$ is an $L$-space knot for any $n \geq-1$, or for any $n \leq 1$.

Following Greene [23, Theorem 1.5], if $K(m)$ is a connected sum of two lens spaces, then $K$ is a torus knot $T_{p, q}$ or a cable of a torus knot $C_{p, q}\left(T_{r, s}\right)$ where $p=q r s \pm 1$. We may assume $p, q \geq 2$ by taking the mirror image if necessary. The next theorem is a refinement of Theorem 1.6(2).

Theorem 1.7 Let $c$ be a seiferter for $(K, m)=\left(T_{p, q}, p q\right)$ or $\left(C_{p, q}\left(T_{r, s}\right), p q\right)$ where $p=q r s \pm 1$. We assume $p, q \geq 2$. Then a knot $K_{n}$ obtained from $K$ by an $n$-twist along $c$ is an $L$-space knot for any $n \geq-1$. Furthermore, if the linking number $l$ between $c$ and $K$ satisfies $l^{2} \geq 2 p q$, then $K_{n}$ is an L-space knot for all integers $n$.

In the above theorem, $K_{n}(n<-1)$ may be an L-space knot even when $l^{2}<2 p q$; see Motegi and Tohki [41].

In Sections 5, 6 and 7 we will exploit seiferter technology developed in Deruelle, Miyazaki and Motegi [13; 12] and Deruelle, Eudave-Muñoz, Miyazaki and Motegi [11] to give a partial answer to Question 1.1. Even though Theorem 1.7 treats a special kind of Seifert surgeries, it offers many applications. In particular, it enables us to give new families of L-space twisted torus knots. See Section 5 for the definition of twisted torus knots $K(p, q ; r, n)$ introduced by Dean [10].

Theorem 1.8 (L-space twisted torus knots)

(1) The following twisted torus knots are $L$-space knots for all integers $n$ :

- $K(p, q ; p+q, n)$ with $p, q \geq 2$,

- $K(3 p+1,2 p+1 ; 4 p+1, n)$ with $p>0$,

- $K(3 p+2,2 p+1 ; 4 p+3, n)$ with $p>0$. 
(2) The following twisted torus knots are $L$-space knots for any $n \geq-1$ :

- $K(p, q ; p-q, n)$ with $p, q \geq 2$,

- $K(2 p+3,2 p+1 ; 2 p+2, n)$ with $p>0$.

Theorem 1.8 has the following corollary, which asserts that every nontrivial torus knot admits twistings desired in Question 1.1.

Corollary 1.9 For any nontrivial torus knot $T_{p, q}$, we can take an unknotted circle $c$ so that an $n$-twist along $c$ converts $T_{p, q}$ into an $L$-space knot $K_{n}$ for all integers $n$. Furthermore, $\left\{K_{n}\right\}_{|n|>3}$ is a set of mutually distinct hyperbolic $L$-space knots.

For the simplest L-space knot, ie the trivial knot $O$, we can strengthen Corollary 1.9 as follows.

Theorem 1.10 (L-space twisted unknots) For the trivial knot $O$, we can take infinitely many unknotted circles $c$ so that an $n$-twist along $c$ changes $O$ into a nontrivial $L$-space knot $K_{c, n}$ for any nonzero integer $n$. Furthermore, $\left\{K_{c, n}\right\}_{|n|>1}$ is a set of mutually distinct hyperbolic L-space knots.

Using a relationship between Berge's lens space surgeries and surgeries yielding a connected sum of two lens spaces, we can prove:

Theorem 1.11 (L-space twisted Berge knots) For any hyperbolic Berge knot $K$, there is an unknotted circle $c$ such that an $n$-twist along $c$ converts $K$ into a hyperbolic $L$-space knot $K_{n}$ for infinitely many integers $n$.

In Section 8, we consider the tunnel number of L-space knots. Recall that the tunnel number of a knot $K$ in $S^{3}$ is the minimum number of mutually disjoint, embedded arcs connecting $K$ such that the exterior of the resulting 1-complex is a handlebody. Hedden's cabling construction [25], together with the work of Morimoto and Sakuma [40], enables us to obtain an L-space knot with tunnel number greater than 1. Actually, Baker and Moore [3] have shown that for any integer $N$, there is an L-space knot with tunnel number greater than $N$. However, the L-space knots with tunnel number greater than one constructed above are all satellite (nonhyperbolic) knots, and they ask:

Question 1.12 [3] Is there a nonsatellite L-space knot with tunnel number greater than one?

Examining knots with Seifert surgeries which do not arise from the primitive/Seifertfibered construction given by Eudave-Muñoz, Jasso, Miyazaki and Motegi [16], we prove the following, which answers the question in the positive. 
Theorem 1.13 There exist infinitely many hyperbolic L-space knots with tunnel number greater than one.

Each knot in the theorem is obtained from a trefoil knot $T_{3,2}$ by alternate twisting along two seiferters for the lens space surgery $\left(T_{3,2}, 7\right)$.

In Section 9, we will discuss further questions on relationships between L-space knots and the twisting operation.

Acknowledgments I would like to thank Ken Baker for insightful and stimulating discussion. This paper would not have come to fruition without examples obtained in previous joint works with Arnaud Deruelle, Mario Eudave-Muñoz, Edgar Jasso and Katura Miyazaki. Finally, I would like to thank the referee for careful reading and useful comments.

The author has been partially supported by JSPS Grants-in-Aid for Scientific Research (C), 26400099, The Ministry of Education, Culture, Sports, Science and Technology, Japan and Joint Research Grant of Institute of Natural Sciences at Nihon University for 2014.

\section{Seifert fibered L-spaces}

Let $M$ be a rational homology 3-sphere which is a Seifert fiber space. For homological reasons, the base surface of $M$ is either $S^{2}$ or $\mathbb{R} P^{2}$. In the latter case, Boyer, Gordon and Watson [8, Proposition 5] prove that $M$ is an L-space. Now assume that the base surface of $M$ is $S^{2}$. Following Ozsváth and Szabó [45, Theorem 1.4], if $M$ is an L-space, then it carries no taut foliation; in particular, it carries no horizontal (ie transverse) foliation. Furthermore, Lisca and Stipsicz [34, Theorem 1.1] prove that the converse also holds. Therefore, a Seifert fibered rational homology 3-sphere $M$ over $S^{2}$ is an L-space if and only if it does not admit a horizontal foliation. Note that if $M$ does not carry a horizontal foliation, then it is necessarily a rational homology 3 -sphere. In fact, if $\left|H_{1}(M ; \mathbb{Z})\right|=\infty$, then $M$ is a surface bundle over the circle (see [27, Theorem VI.34] and [24, page 22]), and hence it has a horizontal foliation. On the other hand, Eisenbud, Hirsh and Neumann [14], Jankins and Neumann [28], and Naimi [42] gave necessary and sufficient conditions for a Seifert fibered 3-manifold to carry a horizontal foliation. Combining them we have Theorem 2.1 below. See also [9, Theorem 5.4]; we follow the convention of Seifert invariants in [9, Section 4].

For ordered triples $\left(a_{1}, a_{2}, a_{3}\right)$ and $\left(b_{1}, b_{2}, b_{3}\right)$, we write $\left(a_{1}, a_{2}, a_{3}\right)<\left(b_{1}, b_{2}, b_{3}\right)$ (resp. $\left.\left(a_{1}, a_{2}, a_{3}\right) \leq\left(b_{1}, b_{2}, b_{3}\right)\right)$ if $a_{i}<b_{i}$ (resp. $\left.a_{i} \leq b_{i}\right)$ for $1 \leq i \leq 3$, and denote 
by $\left(a_{1}, a_{2}, a_{3}\right)^{*}$ the ordered triple $\left(\sigma\left(a_{1}\right), \sigma\left(a_{2}\right), \sigma\left(a_{3}\right)\right)$, where $\sigma$ is a permutation such that $\sigma\left(a_{1}\right) \leq \sigma\left(a_{2}\right) \leq \sigma\left(a_{3}\right)$.

Theorem $2.1 \quad[45 ; 34 ; 14 ; 28 ; 42] \quad$ A Seifert fiber space $S^{2}\left(b, r_{1}, r_{2}, r_{3}\right)$ (for $b \in \mathbb{Z}$ and $\left.0<r_{i}<1\right)$ is an L-space if and only if one of the following holds:

(1) $b \geq 0$ or $b \leq-3$,

(2) $b=-1$ and there are no relatively prime integers $a, k$ such that $0<a \leq k / 2$ and $\left(r_{1}, r_{2}, r_{3}\right)^{*}<(1 / k, a / k,(k-a) / k)$,

(3) $b=-2$ and there are no relatively prime integers $0<a \leq k / 2$ such that $\left(1-r_{1}, 1-r_{2}, 1-r_{3}\right)^{*}<(1 / k, a / k,(k-a) / k)$.

For our purpose, we consider the following problem:

Problem 2.2 Given an integer $b$ and rational numbers $0<r_{1} \leq r_{2}<1$, describe rational numbers $-1 \leq r \leq 1$ for which $S^{2}\left(b, r_{1}, r_{2}, r\right)$ is an L-space.

We begin by observing:

Lemma 2.3 Assume that $0<r_{1} \leq r_{2}<1$.

(1) If $b \geq 0$ or $b \leq-3$, then $S^{2}\left(b, r_{1}, r_{2}, r\right)$ is an L-space for any $0<r<1$.

(2) If $r_{1}+r_{2} \geq 1$, then $S^{2}\left(-1, r_{1}, r_{2}, r\right)$ is an L-space for any $0<r<1$.

(3) If $r_{1}+r_{2} \leq 1$, then $S^{2}\left(-2, r_{1}, r_{2}, r\right)$ is an L-space for any $0<r<1$.

Proof The first assertion is nothing but Theorem 2.1(1).

Suppose for a contradiction that $S^{2}\left(-1, r_{1}, r_{2}, r\right)$ is not an L-space for some $0<r<1$. Then, by Theorem 2.1(2) we can take relatively prime integers $a, k$ with $0<a \leq k / 2$ so that $\left(r_{1}, r_{2}, r\right)^{*}<(1 / k, a / k,(k-a) / k)$. This then implies that $r_{1}<a / k$ and $r_{2}<(k-a) / k$. Hence $r_{1}+r_{2}<a / k+(k-a) / k=1$, a contradiction. This proves (2).

To prove (3), assume for a contradiction that $S^{2}\left(-2, r_{1}, r_{2}, r\right)$ is not an L-space for some $0<r<1$. Then, by Theorem 2.1(3) we have relatively prime integers $a$, $k$ with $0<a \leq k / 2$ such that $\left(1-r_{1}, 1-r_{2}, 1-r\right)^{*}<(1 / k, a / k,(k-a) / k)$. Thus we have $\left(1-r_{2}\right)<a / k$ and $\left(1-r_{1}\right)<(k-a) / k$. Thus $\left(1-r_{1}\right)+\left(1-r_{2}\right)<1$, which implies $r_{1}+r_{2}>1$, contradicting the assumption.

Now let us prove the following, which gives an answer to Problem 2.2. 
Proposition 2.4 Assume that $0<r_{1} \leq r_{2}<1$.

(1) If $b \leq-3$ or $b \geq 1$, then $S^{2}\left(b, r_{1}, r_{2}, r\right)$ is an $L$-space for any $-1 \leq r \leq 1$.

(2) If $b=-2$, then there exists $\varepsilon>0$ such that $S^{2}\left(-2, r_{1}, r_{2}, r\right)$ is an L-space for any $-1 \leq r \leq \varepsilon$. Furthermore, if $r_{1}+r_{2} \leq 1$, then $S^{2}\left(-2, r_{1}, r_{2}, r\right)$ is an $L$-space if $-1 \leq r<1$.

(3) Suppose that $b=-1$.

(i) If $r_{1}+r_{2} \geq 1$, then $S^{2}\left(-1, r_{1}, r_{2}, r\right)$ is an $L$-space for any $0<r \leq 1$.

(ii) If $r_{1}+r_{2} \leq 1$, then $S^{2}\left(-1, r_{1}, r_{2}, r\right)$ is an $L$-space for any $-1 \leq r<0$.

(4) If $b=0$, then there exists $\varepsilon>0$ such that $S^{2}\left(r_{1}, r_{2}, r\right)$ is an $L$-space for any $-\varepsilon \leq r \leq 1$. Furthermore, if $r_{1}+r_{2} \geq 1$, then $S^{2}\left(r_{1}, r_{2}, r\right)$ is an L-space if $-1<r \leq 1$.

Proof If $r=0, \pm 1$, then $S^{2}\left(b, r_{1}, r_{2}, r\right)$ is a lens space.

Claim 2.5 Suppose that $r$ is an integer. Then the lens space $S^{2}\left(b, r_{1}, r_{2}, r\right)$ is $S^{2} \times S^{1}$ if and only if $b+r=-1$ and $r_{1}+r_{2}=1$. In particular, if $b+r \neq-1$, then $S^{2}\left(b, r_{1}, r_{2}, r\right)$ is an L-space.

Proof of claim We recall that $H_{1}\left(S^{2}(a / b, c / d)\right) \cong \mathbb{Z}$ for $b, d \geq 1$ if and only if $a d+b c=0$, ie $a / b+c / d=0$. Thus $S^{2}\left(b, r_{1}, r_{2}, r\right)$ is $S^{2} \times S^{1}$ if and only if $b+r_{1}+r_{2}+r=0$, ie $r_{1}+r_{2}=-b-r \in \mathbb{Z}$. Since $0<r_{i}<1$, we have $r_{1}+r_{2}=1$ and $b+r=-1$.

We divide into two cases, since $0 \leq r \leq 1$ or $-1 \leq r \leq 0$.

Case I $(\mathbf{0} \leq \boldsymbol{r} \leq \mathbf{1})$ (i) If $b \geq 0$ or $b \leq-3$, then $S^{2}\left(b, r_{1}, r_{2}, r\right)$ is an L-space for any $0<r<1$ by Lemma 2.3(1). Since $b+r \neq-1$ for $r=0,1$, by Claim 2.5 $S^{2}\left(b, r_{1}, r_{2}, r\right)$ is an L-space for $r=0,1$. Hence $S^{2}\left(b, r_{1}, r_{2}, r\right)$ is an L-space for any $0 \leq r \leq 1$.

(ii) Suppose that $b=-1$. By Lemma 2.3(2), if $r_{1}+r_{2} \geq 1$, then $S^{2}\left(-1, r_{1}, r_{2}, r\right)$ is an L-space for any $0<r<1$. Since $S^{2}\left(-1, r_{1}, r_{2}, 1\right)$ is an L-space by Claim 2.5, $S^{2}\left(-1, r_{1}, r_{2}, r\right)$ is an L-space for any $0<r \leq 1$.

(iii) Assume $b=-2$. Suppose that $0<r \leq r_{1}$ so that $0<1-r_{2} \leq 1-r_{1} \leq 1-r<1$. Now let $A=\left\{(k-a) / k \mid 1-r_{2}<1 / k, 1-r_{1}<a / k, 0<a \leq k / 2\right.$,

$a$ and $k$ are relatively prime integers $\}$. 
If $A=\varnothing$, ie there are no relatively prime integers $a$ and $k(0<a \leq k / 2)$ such that $1-r_{2}<1 / k$ and $1-r_{1}<a / k$, then $S^{2}\left(-2, r_{1}, r_{2}, r\right)$ is an L-space for any $0<r \leq r_{1}$ by Theorem 2.1. Suppose that $A \neq \varnothing$. Since there are only finitely many integers $k$ satisfying $1-r_{2}<1 / k, A$ consists of only finitely many elements. Let $r_{0}$ be the maximal element in $A$. If $0<r \leq 1-r_{0}$, then $r_{0} \leq 1-r<1$, and hence there are no relatively prime integers $a$ and $k$ satisfying both

$$
0<a \leq k / 2 \quad \text { and } \quad\left(1-r_{2}, 1-r_{1}, 1-r\right)<(1 / k, a / k,(k-a) / k) .
$$

Let $\varepsilon=\min \left\{r_{1}, 1-r_{0}\right\}$. Then $S^{2}\left(-2, r_{1}, r_{2}, r\right)$ is an L-space for any $0<r \leq \varepsilon$ by Theorem 2.1. Since $S^{2}\left(-2, r_{1}, r_{2}, 0\right)$ is an L-space by Claim $2.5, S^{2}\left(-2, r_{1}, r_{2}, r\right)$ is an L-space for any $0 \leq r \leq \varepsilon$. Furthermore, if we have the additional condition $r_{1}+r_{2} \leq 1$, then Lemma 2.3(3) improves the result so that $S^{2}\left(-2, r_{1}, r_{2}, r\right)$ is an L-space for any $0 \leq r<1$.

Case II $(-\mathbf{1} \leq \boldsymbol{r} \leq \mathbf{0}) \quad$ Note that $S^{2}\left(b, r_{1}, r_{2}, r\right)=S^{2}\left(b-1, r_{1}, r_{2}, r+1\right)$.

(i) If $b \geq 1$ or $b \leq-2$ (ie $b-1 \geq 0$ or $b-1 \leq-3$ ), then $S^{2}\left(b, r_{1}, r_{2}, r\right)=$ $S^{2}\left(b-1, r_{1}, r_{2}, r+1\right)$ is an L-space for any $0<r+1<1$, ie $-1<r<0$, by Lemma 2.3(1). Since $b+r \neq-1$ for $r=-1,0$, then $S^{2}\left(b, r_{1}, r_{2}, r\right)$ is an L-space for $r=-1,0$ by Claim 2.5. Thus $S^{2}\left(b, r_{1}, r_{2}, r\right)$ is an L-space for any $-1 \leq r \leq 0$.

(ii) If $b=0$ (ie $b-1=-1)$, then $S^{2}\left(0, r_{1}, r_{2}, r\right)=S^{2}\left(-1, r_{1}, r_{2}, r+1\right)$. Let us assume $r_{2}-1 \leq r<0$ so that $0<r_{1} \leq r_{2} \leq r+1<1$. Set

$A=\left\{(k-a) / k \mid r_{1}<1 / k, r_{2}<a / k, 0<a \leq k / 2\right.$,

$a$ and $k$ are relatively prime integers $\}$.

If $A=\varnothing$, for any $r$ with $r_{2} \leq r+1<1$, we can easily observe that $S^{2}\left(-1, r_{1}, r_{2}, r+1\right)$ is an L-space by Theorem 2.1. Hence for any $r_{2}-1 \leq r<0, S^{2}\left(0, r_{1}, r_{2}, r\right)$ is an L-space. Suppose that $A \neq \varnothing$. Since $A$ is a finite set, we take the maximal element $r_{0}$ in $A$. If $r_{0} \leq r+1<1$ (ie $r_{0}-1 \leq r<0$ ), then there are no relatively prime integers $a$ and $k$ satisfying both

$$
0<a \leq k / 2 \quad \text { and } \quad\left(r_{1}, r_{2}, r+1\right)<(1 / k, a / k,(k-a) / k) .
$$

Let $\varepsilon=\min \left\{1-r_{2}, 1-r_{0}\right\}$. Then $S^{2}\left(0, r_{1}, r_{2}, r\right)=S^{2}\left(-1, r_{1}, r_{2}, r+1\right)$ is an L-space for any $-\varepsilon \leq r<0$ by Theorem 2.1. Since $S^{2}\left(0, r_{1}, r_{2}, 0\right)=S^{2}\left(r_{1}, r_{2}\right)$ is an L-space by Claim 2.5, $S^{2}\left(0, r_{1}, r_{2}, r\right)$ is an L-space for any $-\varepsilon \leq r \leq 0$. Furthermore, if we have the additional condition $r_{1}+r_{2} \geq 1$, then Lemma 2.3(2) improves the result so that $S^{2}\left(r_{1}, r_{2}, r\right)=S^{2}\left(-1, r_{1}, r_{2}, r+1\right)$ is an L-space for any $-1<r \leq 0$.

(iii) If $b=-1$ (ie $b-1=-2$ ), then $S^{2}\left(-1, r_{1}, r_{2}, r\right)=S^{2}\left(-2, r_{1}, r_{2}, r+1\right)$. Assume that $r_{1}+r_{2} \leq 1$. By Lemma 2.3(3), $S^{2}\left(-1, r_{1}, r_{2}, r\right)=S^{2}\left(-2, r_{1}, r_{2}, r+1\right)$ 
is an L-space for any $0<r+1<1$, ie $-1<r<0$. Since Claim 2.5 shows that $S^{2}\left(-1, r_{1}, r_{2},-1\right)$ is an L-space, $S^{2}\left(-1, r_{1}, r_{2}, r\right)$ is an L-space for any $-1 \leq r<0$.

Combining cases I and II, the proof of Proposition 2.4 is complete.

The next proposition shows that if $S^{2}\left(b, r_{1}, r_{2}, r_{\infty}\right)$ is an L-space for some rational number $0<r_{\infty}<1$, then we can find $r$ near $r_{\infty}$ such that $S^{2}\left(b, r_{1}, r_{2}, r\right)$ is an L-space.

Proposition 2.6 Suppose that $0<r_{1} \leq r_{2}<1$ and $S^{2}\left(b, r_{1}, r_{2}, r_{\infty}\right)$ is an L-space for some rational number $0<r_{\infty}<1$.

(1) If $b=-1$, then $S^{2}\left(-1, r_{1}, r_{2}, r\right)$ is an $L$-space for any $r_{\infty} \leq r \leq 1$.

(2) If $b=-2$, then $S^{2}\left(-2, r_{1}, r_{2}, r\right)$ is an $L$-space for any $-1 \leq r \leq r_{\infty}$.

Proof (1) Assume for a contradiction that $S^{2}\left(-1, r_{1}, r_{2}, r\right)$ is not an L-space for some $r$ satisfying $r_{\infty} \leq r<1$. By Theorem 2.1 we have relatively prime integers $a$ and $k$ with $0<a \leq k / 2$ such that $\left(r_{1}, r_{2}, r\right)^{*}<(1 / k, a / k,(k-a) / k)$. Since we have $r_{\infty} \leq r<1$, it follows that

$$
\left(r_{1}, r_{2}, r_{\infty}\right)^{*} \leq\left(r_{1}, r_{2}, r\right)^{*}<(1 / k, a / k,(k-a) / k) .
$$

Hence Theorem 2.1 shows that $S^{2}\left(-1, r_{1}, r_{2}, r_{\infty}\right)$ is not an L-space, a contradiction. Since $S^{2}\left(-1, r_{1}, r_{2}, 1\right)=S^{2}\left(r_{1}, r_{2}\right)$ is an L-space by Claim $2.5, S^{2}\left(-1, r_{1}, r_{2}, r\right)$ is an L-space for any $r_{\infty} \leq r \leq 1$.

(2) Next assume for a contradiction that $S^{2}\left(-2, r_{1}, r_{2}, r\right)$ is not an L-space for some $r$ satisfying $0<r \leq r_{\infty}$. Then following Theorem 2.1 we have $\left(1-r_{1}, 1-r_{2}, 1-r\right)^{*}<$ $(1 / k, a / k,(k-a) / k)$ for some relatively prime integers $a$ and $k$ with $0<a \leq k / 2$. Since $r \leq r_{\infty}$, we have $1-r_{\infty} \leq 1-r$, and hence

$$
\begin{aligned}
\left(1-r_{1}, 1-r_{2}, 1-r_{\infty}\right)^{*} & \leq\left(1-r_{1}, 1-r_{2}, 1-r\right)^{*} \\
& <(1 / k, a / k,(k-a) / k) .
\end{aligned}
$$

This means $S^{2}\left(-2, r_{1}, r_{2}, r_{\infty}\right)$ is not an L-space, contradicting the assumption. Thus $S^{2}\left(-2, r_{1}, r_{2}, r\right)$ is an L-space for any $0<r \leq r_{\infty}$. Furthermore, as shown in Proposition 2.4(2), $S^{2}\left(-2, r_{1}, r_{2}, r\right)$ is an L-space if $-1 \leq r \leq \varepsilon$ for some $\varepsilon>0$, so $S^{2}\left(-2, r_{1}, r_{2}, r\right)$ is an L-space for any $-1 \leq r \leq r_{\infty}$.

We close this section with the complement of Proposition 2.6. 
Proposition 2.7 Suppose that $0<r_{1} \leq r_{2}<1$ and $S^{2}\left(b, r_{1}, r_{2}, r_{\infty}\right)$ is not an L-space for some rational number $0<r_{\infty}<1$.

(1) If $b=-1$, then there exists $\varepsilon>0$ such that $S^{2}\left(-1, r_{1}, r_{2}, r\right)$ is not an $L$-space for any $0<r<r_{\infty}+\varepsilon$.

(2) If $b=-2$, then there exists $\varepsilon>0$ such that then $S^{2}\left(-2, r_{1}, r_{2}, r\right)$ is an $L$-space for any $r_{\infty}-\varepsilon<r<1$.

Proof (1) Since $S^{2}\left(-1, r_{1}, r_{2}, r_{\infty}\right)$ is not an L-space, Theorem 2.1 shows that there are relatively prime integers $a$ and $k$ with $0<a \leq k / 2$ such that $\left(r_{1}, r_{2}, r_{\infty}\right)^{*}<$ $(1 / k, a / k,(k-a) / k)$. Then there exists $\varepsilon>0$ such that for any $0<r<r_{\infty}+\varepsilon$, we have $\left(r_{1}, r_{2}, r\right)^{*}<(1 / k, a / k,(k-a) / k)$. Thus by Theorem 2.1 again, $S^{2}\left(-1, r_{1}, r_{2}, r\right)$ is not an L-space for any $0<r<r_{\infty}+\varepsilon$.

(2) Since $S^{2}\left(-2, r_{1}, r_{2}, r_{\infty}\right)$ is not an L-space, by Theorem 2.1 we have relatively prime integers $a$ and $k$ such that

$$
0<a \leq k / 2 \quad \text { and } \quad\left(1-r_{1}, 1-r_{2}, 1-r_{\infty}\right)^{*}<(1 / k, a / k,(k-a) / k) .
$$

Hence there exists $\varepsilon>0$ such that if $0<1-r<1-r_{\infty}+\varepsilon$, ie $r_{\infty}-\varepsilon<r<1$, then $\left(1-r_{1}, 1-r_{2}, 1-r\right)^{*}<(1 / k, a / k,(k-a) / k)$. By Theorem 2.1, $S^{2}\left(-2, r_{1}, r_{2}, r\right)$ is not an L-space for any $r_{\infty}-\varepsilon<r<1$.

\section{L-space surgeries and twisting along seiferters, I: Nondegenerate case}

The goal in this section is to prove Theorems 1.4 and 1.5.

Let $c$ be a seiferter for a small Seifert fibered surgery $(K, m)$. The 3-manifold obtained by $(m, 0)$-surgery on $K \cup c$ is denoted by $M_{c}(K, m)$.

Proof of Theorem 1.4 First we prove the "if" part. If $K(m)$ is a lens space and $c$ is a core of the genus-one Heegaard splitting, then $K_{n}\left(m_{n}\right)$ is a lens space for any integer $n$. Thus $\left(K_{n}, m_{n}\right)$ is an L-space surgery for all $n \in \mathbb{Z}$ except when $K_{n}\left(m_{n}\right) \cong S^{2} \times S^{1}$, ie $K_{n}$ is the trivial knot and $m_{n}=0$; see [17, Theorem 8.1]. Since $\left(K_{n}, m_{n}\right)=\left(K_{n^{\prime}}, m_{n^{\prime}}\right)$ if and only if $n=n^{\prime}$ by [13, Theorem 5.1], there is at most one integer $n$ such that $\left(K_{n}, m_{n}\right)=(O, 0)$. Henceforth, in the case where $K(m)$ is a lens space, we assume that $K(m)$ has a Seifert fibration over $S^{2}$ with two exceptional fibers $t_{1}$ and $t_{2}$, and $c$ becomes a regular fiber in this Seifert fibration.

Let $E$ be $K(m)-$ int $N(c)$ with a fibered tubular neighborhood of the union of two exceptional fibers $t_{1}$ and $t_{2}$ and one regular fiber $t_{0}$ removed. Then $E$ is a product 
circle bundle over the fourth-punctured sphere. Take a cross section of $E$ such that $K(m)$ is expressed as $S^{2}\left(b, r_{1}, r_{2}, r_{3}\right)$, where the Seifert invariant of $t_{0}$ is $b \in \mathbb{Z}$, that of $t_{i}$ is $0<r_{i}<1$ for $i=1,2$, and that of $c$ is $0 \leq r_{3}<1$. Without loss of generality, we may assume $r_{1} \leq r_{2}$. Let $s$ be the boundary curve on $\partial N(c)$ of the cross section so that $[s] \cdot[t]=1$ for a regular fiber $t \subset \partial N(c)$. Let $(\mu, \lambda)$ be a preferred meridian-longitude pair of $c \subset S^{3}$. Then

$$
[\mu]=\alpha_{3}[s]+\beta_{3}[t] \in H_{1}(\partial N(c)) \quad \text { and } \quad[\lambda]=-\alpha[s]-\beta[t] \in H_{1}(\partial N(c))
$$

for some integers $\alpha_{3}, \beta_{3}, \alpha$ and $\beta$ which satisfy $\alpha_{3}>0$ and $\alpha \beta_{3}-\beta \alpha_{3}=1$, where $r_{3}=\beta_{3} / \alpha_{3}$. Now let us write $r_{c}=\beta / \alpha$, which is the slope of the preferred longitude $\lambda$ of $c \subset S^{3}$ with respect to the $(s, t)$-basis.

Claim 3.1 $M_{c}(K, m)$ is a (possibly degenerate) Seifert fiber space $S^{2}\left(b, r_{1}, r_{2}, r_{c}\right)$; if $r_{c}=-1 / 0$, then it is a connected sum of two lens spaces.

Proof of claim $M_{c}(K, m)$ is regarded as a 3-manifold obtained from $K(m)$ by performing $\lambda$-surgery along the fiber $c \subset K(m)$. Since $[\lambda]=-\alpha[s]-\beta[t]$, we see that $M_{c}(K, m)$ is a (possibly degenerate) Seifert fiber space $S^{2}\left(b, r_{1}, r_{2}, r_{c}\right)$. If $\alpha=0$, ie $r_{c}=-1 / 0$, then $M_{c}(K, m)$ has a degenerate Seifert fibration, and it is a connected sum of two lens spaces.

Recall that $\left(K_{n}, m_{n}\right)$ is a Seifert surgery obtained from $(K, m)$ by twisting $n$ times along $c$. The image of $c$ after the $n$-twist along $c$ is also a seiferter for $\left(K_{n}, m_{n}\right)$, and is denoted by $c_{n}$. We study how the Seifert invariant of $K(m)$ behaves under the twisting. We compute the Seifert invariant of $c_{n}$ in $K_{n}\left(m_{n}\right)$ under the same cross section on $E$.

Since we have

$$
\left(\begin{array}{l}
{[\mu]} \\
{[\lambda]}
\end{array}\right)=\left(\begin{array}{cc}
\alpha_{3} & \beta_{3} \\
-\alpha & -\beta
\end{array}\right)\left(\begin{array}{l}
{[s]} \\
{[t]}
\end{array}\right),
$$

it follows that

$$
\left(\begin{array}{l}
{[s]} \\
{[t]}
\end{array}\right)=\left(\begin{array}{rr}
-\beta & -\beta_{3} \\
\alpha & \alpha_{3}
\end{array}\right)\left(\begin{array}{l}
{[\mu]} \\
{[\lambda]}
\end{array}\right) .
$$

Twisting $n$ times along $c$ is equivalent to performing $-1 / n$-surgery on $c$. A preferred meridian-longitude pair $\left(\mu_{n}, \lambda_{n}\right)$ of $N\left(c_{n}\right) \subset S^{3}$ satisfies $\left[\mu_{n}\right]=[\mu]-n[\lambda]$ and $\left[\lambda_{n}\right]=[\lambda]$ in $H_{1}\left(\partial N\left(c_{n}\right)\right)=H_{1}(\partial N(c))$.

We thus have

$$
\left(\begin{array}{l}
{[s]} \\
{[t]}
\end{array}\right)=\left(\begin{array}{rr}
-\beta & -n \beta-\beta_{3} \\
\alpha & n \alpha+\alpha_{3}
\end{array}\right)\left(\begin{array}{l}
{\left[\mu_{n}\right]} \\
{\left[\lambda_{n}\right]}
\end{array}\right),
$$


and it follows that

$$
\left(\begin{array}{l}
{\left[\mu_{n}\right]} \\
{\left[\lambda_{n}\right]}
\end{array}\right)=\left(\begin{array}{cc}
n \alpha+\alpha_{3} & n \beta+\beta_{3} \\
-\alpha & -\beta
\end{array}\right)\left(\begin{array}{l}
{[s]} \\
{[t]}
\end{array}\right) .
$$

Hence the Seifert invariant of the fiber $c_{n}$ in $K_{n}\left(m_{n}\right)$ is $\left(n \beta+\beta_{3}\right) /\left(n \alpha+\alpha_{3}\right)$, and $K_{n}\left(m_{n}\right)=S^{2}\left(b, r_{1}, r_{2},\left(n \beta+\beta_{3}\right) /\left(n \alpha+\alpha_{3}\right)\right)$.

Remark 3.2 Since $\left(n \beta+\beta_{3}\right) /\left(n \alpha+\alpha_{3}\right)$ converges to $\beta / \alpha$ when $|n|$ tends to $\infty$, $M_{c}(K, m)$ can be regarded as the limit of $K_{n}\left(m_{n}\right)$ when $|n|$ tends to $\infty$.

We divide into three cases: $r_{c}=-1 / 0, r_{c} \in \mathbb{Z}$ or $r_{c} \in \mathbb{Q} \backslash \mathbb{Z}$. Except for the last case, we do not need the assumption that $M_{c}(K, m)$ is an L-space.

Case 1 Suppose that $r_{c}=\beta / \alpha=-1 / 0$. Since $\alpha_{3}>0$ and $\alpha \beta_{3}-\beta \alpha_{3}=1$, we have $\alpha_{3}=1$ and $\beta=-1$. Hence $K_{n}\left(m_{n}\right)$ is a Seifert fiber space:

$$
K_{n}\left(m_{n}\right)=S^{2}\left(b, r_{1}, r_{2},\left(n \beta+\beta_{3}\right) /\left(n \alpha+\alpha_{3}\right)\right)=S^{2}\left(b, r_{1}, r_{2},-n+\beta_{3}\right),
$$

which is a lens space for any $n \in \mathbb{Z}$. Following Claim $2.5, S^{2}\left(b, r_{1}, r_{2},-n+\beta_{3}\right)$ is an L-space if $n \neq b+\beta_{3}+r_{1}+r_{2}$. Thus $\left(K_{n}, m_{n}\right)$ is an L-space surgery for all $n \in \mathbb{Z}$ except possibly $n=b+\beta_{3}+r_{1}+r_{2}$.

Next suppose that $r_{c}=\beta / \alpha \neq-1 / 0$. Then the Seifert invariant of $c_{n}$ is

$$
f(n)=\frac{n \beta+\beta_{3}}{n \alpha+\alpha_{3}}=\frac{\beta}{\alpha}+\frac{\beta_{3}-(\beta / \alpha) \alpha_{3}}{n \alpha+\alpha_{3}}=r_{c}+\frac{\beta_{3}-r_{c} \alpha_{3}}{n \alpha+\alpha_{3}} .
$$

Since $\alpha \beta_{3}-\beta \alpha_{3}=\alpha\left(\beta_{3}-r_{c} \alpha_{3}\right)=1$, then $\alpha$ and $\beta_{3}-r_{c} \alpha_{3}$ have the same sign.

Case 2 Now suppose that $r_{c} \in \mathbb{Z}$. Let $p=r_{c}$. Then we can write $S^{2}\left(b, r_{1}, r_{2}, r_{c}\right)=$ $S^{2}\left(b+p, r_{1}, r_{2}\right)$.

(i) If we have $b \leq-p-3$ or $b \geq-p+1$, then Proposition 2.4(1) shows that $S^{2}\left(b, r_{1}, r_{2}, f(n)\right)=S^{2}\left(b+p, r_{1}, r_{2}, f(n)-p\right)$ is an L-space if $-1 \leq f(n)-p \leq 1$, ie $p-1 \leq f(n) \leq p+1$. Hence $\left(K_{n}, m_{n}\right)$ is an L-space for all $n$ but $n \in\left(x_{1}, x_{2}\right)$, where $f\left(x_{1}\right)=p-1$ and $f\left(x_{2}\right)=p+1$; see Figure 2(left).

(ii) If $b=-p-2$, then it follows from Proposition 2.4(2) that there is an $\varepsilon>0$ such that $S^{2}\left(b, r_{1}, r_{2}, f(n)\right)=S^{2}\left(b+p, r_{1}, r_{2}, f(n)-p\right)=S^{2}\left(-2, r_{1}, r_{2}, f(n)-p\right)$ is an L-space if $-1 \leq f(n)-p \leq \varepsilon$. Hence $\left(K_{n}, m_{n}\right)$ is an L-space except for only finitely many $n \in\left(x_{1}, x_{2}\right)$, where $f\left(x_{1}\right)=p-1, f\left(x_{2}\right)=p+\varepsilon$; see Figure 2(middle).

(iii) Suppose that $b=-p-1$. If $r_{1}+r_{2} \geq 1$ (resp. $r_{1}+r_{2} \leq 1$ ), then Proposition 2.4(3) shows that $S^{2}\left(b, r_{1}, r_{2}, f(n)\right)=S^{2}\left(b+p, r_{1}, r_{2}, f(n)-p\right)=S^{2}\left(-1, r_{1}, r_{2}, f(n)-p\right)$ is an L-space if $0<f(n)-p \leq 1$ (resp. $-1 \leq f(n)-p<0)$. Hence $\left(K_{n}, m_{n}\right)$ is 


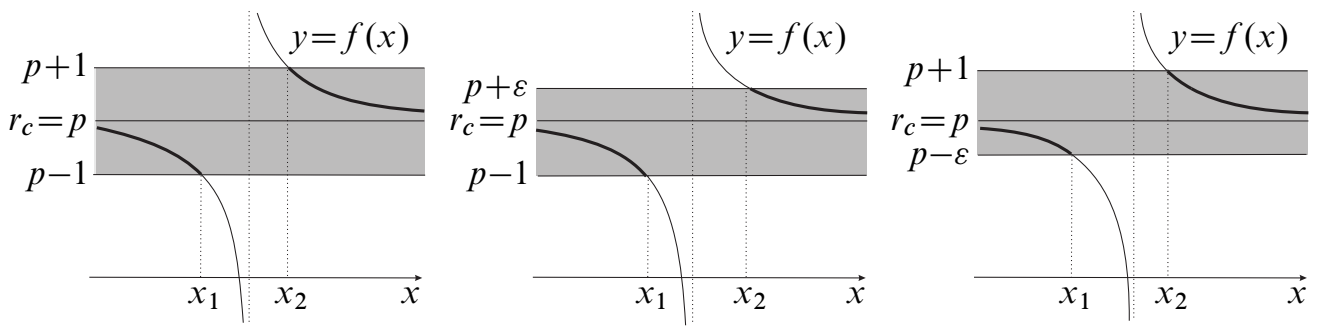

Figure 2: $f(x)=\frac{\beta x+\beta_{3}}{\alpha x+\alpha_{3}}$.

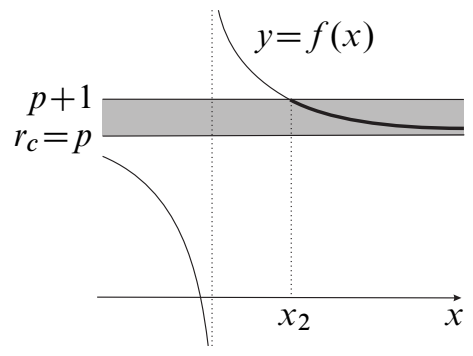

$r_{1}+r_{2} \geq 1$

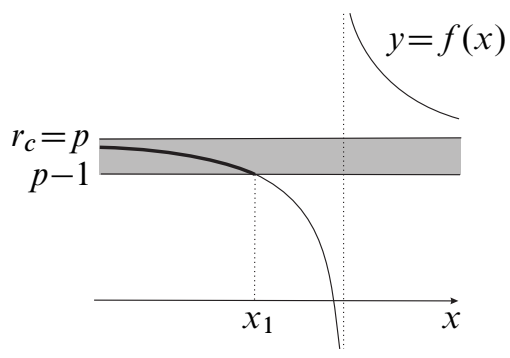

$r_{1}+r_{2} \leq 1$

Figure 3: $f(x)=\frac{\beta x+\beta_{3}}{\alpha x+\alpha_{3}}$.

an L-space for any integer $n \geq x_{2}$, where $f\left(x_{2}\right)=p+1$ (resp. $n \leq x_{1}$, where $\left.f\left(x_{1}\right)=p-1\right)$, see Figure 3 .

(iv) Suppose that $b=-p$. Then Proposition 2.4(4) shows that

$$
S^{2}\left(b, r_{1}, r_{2}, f(n)\right)=S^{2}\left(b+p, r_{1}, r_{2}, f(n)-p\right)=S^{2}\left(r_{1}, r_{2}, f(n)-p\right)
$$

is an L-space if $-\varepsilon \leq f(n)-p \leq 1$, ie $p-\varepsilon \leq f(n) \leq p+1$, for some $\varepsilon>0$. Hence $\left(K_{n}, m_{n}\right)$ is an L-space for all $n$ but $n \in\left(x_{1}, x_{2}\right)$, where $f\left(x_{1}\right)=p-\varepsilon$ and $f\left(x_{2}\right)=p+1$; see Figure 2(right).

Case 3 Finally, suppose that $r_{c} \in \mathbb{Q} \backslash \mathbb{Z}$ and that $M_{c}(K, m)=S^{2}\left(b, r_{1}, r_{2}, r_{c}\right)$ is an L-space. We assume $p<r_{c}<p+1$ for some integer $p$. Then we have $S^{2}\left(b, r_{1}, r_{2}, r_{c}\right)=S^{2}\left(b+p, r_{1}, r_{2}, r_{c}-p\right)$, where $0<r_{c}-p<1$.

(i) If $b \leq-p-3$ or $b \geq-p+1$, then by Proposition 2.4(1), $S^{2}\left(b, r_{1}, r_{2}, f(n)\right)=$ $S^{2}\left(b+p, r_{1}, r_{2}, f(n)-p\right)$ is an L-space if $-1 \leq f(n)-p \leq 1$, ie $p-1 \leq f(n) \leq p+1$. Hence $\left(K_{n}, m_{n}\right)$ is an L-space for all $n$ but $n \in\left(x_{1}, x_{2}\right)$, where $f\left(x_{1}\right)=p-1$ and $f\left(x_{2}\right)=p+1 ;$ see Figure 4(left). 

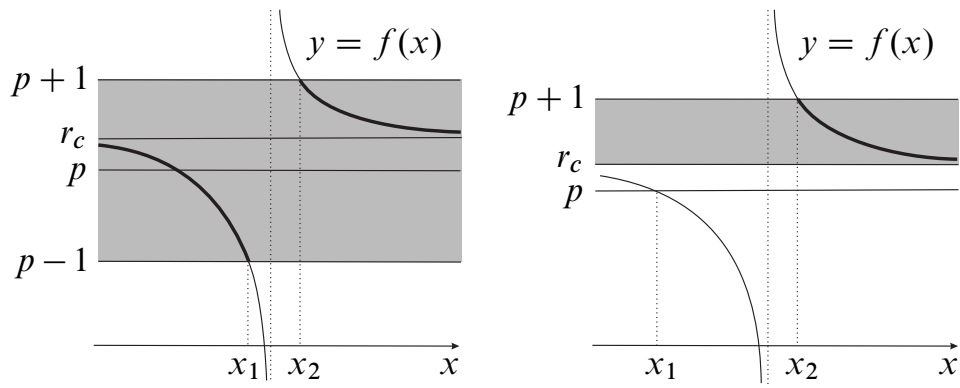

Figure 4: $f(x)=\frac{\beta x+\beta_{3}}{\alpha x+\alpha_{3}}$.
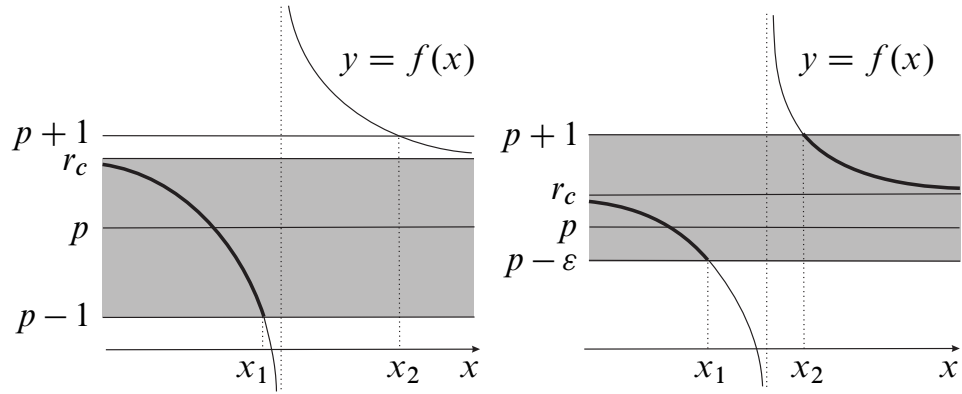

Figure 5: $f(x)=\frac{\beta x+\beta_{3}}{\alpha x+\alpha_{3}}$.

(ii) Suppose that $b=-p-1$. Since

$$
S^{2}\left(b, r_{1}, r_{2}, r_{c}\right)=S^{2}\left(b+p, r_{1}, r_{2}, r_{c}-p\right)=S^{2}\left(-1, r_{1}, r_{2}, r_{c}-p\right)
$$

is an L-space, by Proposition 2.6(1), $S^{2}\left(b, r_{1}, r_{2}, f(n)\right)=S^{2}\left(-1, r_{1}, r_{2}, f(n)-p\right)$ is an L-space if $r_{c}-p \leq f(n)-p \leq 1$, ie $r_{c} \leq f(n) \leq p+1$. Hence $\left(K_{n}, m_{n}\right)$ is an L-space for any $n \geq x_{2}$, where $f\left(x_{2}\right)=p+1$; see Figure 4(right). (Furthermore, if $r_{1}+r_{2} \geq 1$, then by Proposition 2.4(3i), $S^{2}\left(-1, r_{1}, r_{2}, f(n)-p\right)$ is an L-space provided $0<f(n)-p \leq 1$, ie $p<f(n) \leq p+1$. Hence $\left(K_{n}, m_{n}\right)$ is an L-space surgery for any integer $n$ except for $n \in\left[x_{1}, x_{2}\right)$, where $f\left(x_{1}\right)=p$ and $f\left(x_{2}\right)=p+1$.)

(iii) Suppose that $b=-p-2$. Since

$$
S^{2}\left(b, r_{1}, r_{2}, r_{c}\right)=S^{2}\left(b+p, r_{1}, r_{2}, r_{c}-p\right)=S^{2}\left(-2, r_{1}, r_{2}, r_{c}-p\right)
$$

is an L-space, by Proposition 2.6(2), $S^{2}\left(b, r_{1}, r_{2}, f(n)\right)=S^{2}\left(-2, r_{1}, r_{2}, f(n)-p\right)$ is an L-space if $-1 \leq f(n)-p \leq r_{c}-p$, ie $p-1 \leq f(n) \leq r_{c}$. Hence $\left(K_{n}, m_{n}\right)$ is an L-space for $n \leq x_{1}$, where $f\left(x_{1}\right)=p-1$; see Figure 5(left). (Furthermore, if $r_{1}+r_{2} \leq 1$, then by Proposition 2.4(2), $S^{2}\left(-2, r_{1}, r_{2}, f(n)-p\right)$ is an L-space 
provided $-1 \leq f(n)-p<1$, ie $p-1 \leq f(n)<p+1$. Hence $\left(K_{n}, m_{n}\right)$ is an L-space surgery for any integer $n$ except for $n \in\left(x_{1}, x_{2}\right]$, where $f\left(x_{1}\right)=p-1$ and $f\left(x_{2}\right)=p+1$.)

(iv) If $b=-p$, then it follows from Proposition 2.4(4) that

$$
S^{2}\left(b, r_{1}, r_{2}, f(n)\right)=S^{2}\left(b+p, r_{1}, r_{2}, f(n)-p\right)=S^{2}\left(r_{1}, r_{2}, f(n)-p\right)
$$

is an L-space if $-\varepsilon \leq f(n)-p \leq 1$, ie $p-\varepsilon \leq f(n) \leq p+1$, for some $\varepsilon>0$. Hence $\left(K_{n}, m_{n}\right)$ is an L-space for all $n$ but $n \in\left(x_{1}, x_{2}\right)$, where $f\left(x_{1}\right)=p-\varepsilon$ and $f\left(x_{2}\right)=p+1$; see Figure 5(right).

Now let us prove the "only if" part of Theorem 1.4. We begin by observing:

Lemma 3.3 $M_{c}(K, m)$ cannot be $S^{2} \times S^{1}$; in particular, if $M_{c}(K, m)$ is a lens space, then it is an L-space.

Proof Let $w$ be the linking number between $c$ and $K$. Then $H_{1}\left(M_{c}(K, m)\right)=$ $\left\langle\mu_{c}, \mu_{K} \mid w \mu_{c}+m \mu_{K}=0, w \mu_{K}=0\right\rangle$, where $\mu_{c}$ is a meridian of $c$ and $\mu_{K}$ is that of $K$. If $M_{c}(K, m) \cong S^{2} \times S^{1}$, then $H_{1}\left(M_{c}(K, m)\right) \cong \mathbb{Z}$, and we have $w=0$. Let $V=S^{3}-$ int $N(c)$, which is a solid torus containing $K$ in its interior; $K$ is not contained in any 3-ball in $V$. Since $w=0$, we know $K$ is null-homologous in $V$. Furthermore, since $c$ is a seiferter for $(K, m)$, the result $V(K ; m)$ of $V$ after $m$-surgery on $K$ has a (possibly degenerate) Seifert fibration. Then [13, Lemma 3.22] shows that the Seifert fibration of $V(K ; m)$ is nondegenerate and neither a meridian nor a longitude of $V$ is a fiber in $V(K ; m)$, and the base surface of $V(K ; m)$ is not a Möbius band. Since $K$ is null-homologous in $V$, we know $V(K ; m)$ is not a solid torus [18, Theorem 1.1], and hence $V(K ; m)$ has a Seifert fibration over the disk with at least two exceptional fibers. Then $M_{c}(K, m)=V(K ; m) \cup N(c)$ is obtained by attaching $N(c)$ to $V(K ; m)$ so that the meridian of $N(c)$ is identified with a meridian of $V$. Since a regular fiber on $\partial V(K ; m)$ intersects a meridian of $V$ (ie a meridian of $N(c))$ more than once, $M_{c}(K, m)$ is a Seifert fiber space over $S^{2}$ with at least three exceptional fibers. Therefore $M_{c}(K, m)$ cannot be $S^{2} \times S^{1}$. Thus the lemma is proved.

Suppose first that $K(m)$ is a lens space and $c$ is a core of a genus-one Heegaard splitting of $K(m)$. Then $V(K ; m)=K(m)-$ int $N(c)$ is a solid torus, and $M_{c}(K, m)=$ $V(K ; m) \cup N(c)$ is obviously a lens space. By Lemma 3.3, $M_{c}(K, m)$ is an L-space.

In the remaining case, as in the proof of the "if" part of Theorem 1.4, $M_{c}(K, m)$ has the form $S^{2}\left(b, r_{1}, r_{2}, r_{c}\right)$, where $0<r_{1} \leq r_{2}<1$. 


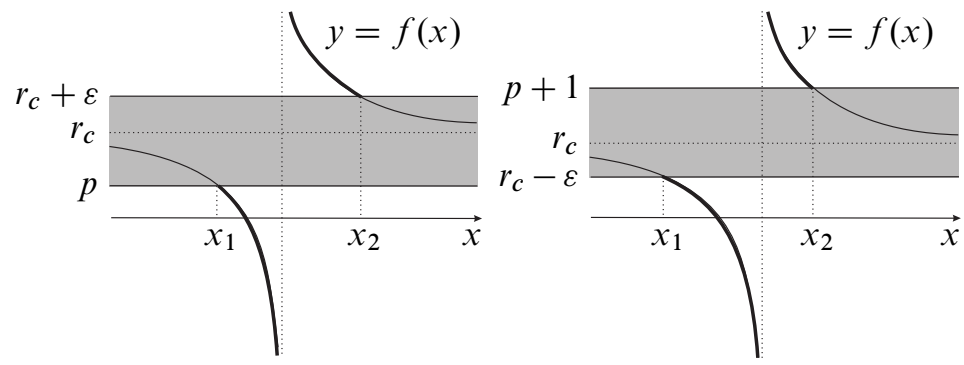

Figure 6: $f(x)=\frac{\beta x+\beta_{3}}{\alpha x+\alpha_{3}}$.

Claim 3.4 If $r_{c}=-1 / 0$ or $r_{c} \in \mathbb{Z}$, then $M_{c}(K, m)$ is an $L$-space.

Proof of claim If $r_{c}=-1 / 0$, then $M_{c}(K, m)=S^{2}\left(b, r_{1}, r_{2},-1 / 0\right)$ is a connected sum of two lens spaces. Since a connected sum of L-spaces is also an L-space [50, page 221] (see also [46, Proposition 6.1]), $M_{c}(K, m)$ is an L-space. If $r_{c} \in \mathbb{Z}$, then $M_{c}(K, m)$ is a lens space; hence it is an L-space by Lemma 3.3.

Now suppose that $M_{c}(K, m)$ is not an L-space. By Claim 3.4, $r_{c} \in \mathbb{Q} \backslash \mathbb{Z}$. We write $r_{c}=r_{c}^{\prime}+p$ so that $0<r_{c}^{\prime}<1$ and $p \in \mathbb{Z}$. Then $M_{c}(K, m)=S^{2}\left(b, r_{1}, r_{2}, r_{c}\right)=$ $S^{2}\left(b+p, r_{1}, r_{2}, r_{c}^{\prime}\right)$. Since $M_{c}(K, m)$ is not an L-space, $b+p=-1$ or -2 by Theorem 2.1. It follows from Proposition 2.7 that there is an $\varepsilon>0$ such that

$$
\begin{aligned}
K_{n}\left(m_{n}\right) & =S^{2}\left(b, r_{1}, r_{2}, f(n)\right) \\
& =S^{2}\left(b+p, r_{1}, r_{2}, f(n)-p\right) \\
& =S^{2}\left(-1, r_{1}, r_{2}, f(n)-p\right) \quad\left(\text { resp. } S^{2}\left(-2, r_{1}, r_{2}, f(n)-p\right)\right)
\end{aligned}
$$

is not an L-space if $0<f(n)-p<r_{c}^{\prime}+\varepsilon$, ie $p<f(n)<r_{c}+\varepsilon$ (resp. $r_{c}^{\prime}-\varepsilon<$ $f(n)-p<1$, ie $\left.r_{c}-\varepsilon<f(n)<p+1\right)$. Hence there are at most finitely many integers $n$ such that $K_{n}\left(m_{n}\right)$ is an L-space, ie $\left(K_{n}, m_{n}\right)$ is an L-space surgery. See Figure 6.

This completes the proof of Theorem 1.4.

Proof of Theorem 1.5 Note that either $K_{n}\left(m_{n}\right)$ is a Seifert fiber space which admits a Seifert fibration over $\mathbb{R} P^{2}$, or $K_{n}\left(m_{n}\right)$ has $S^{2} \times S^{1}$ as a connected summand, depending on whether $c$ becomes a nondegenerate fiber or a degenerate fiber in $K_{n}\left(m_{n}\right)$, respectively. In the former case, Boyer, Gordon and Watson [8, Proposition 5] prove that $K_{n}\left(m_{n}\right)$ is an L-space. In the latter case, $\left(K_{n}, m_{n}\right)=(O, 0)$ (see [17, Theorem 8.1]), which is not an L-space surgery, but there is at most one such integer $n$; see [13, Theorem 5.1]. This completes the proof. 


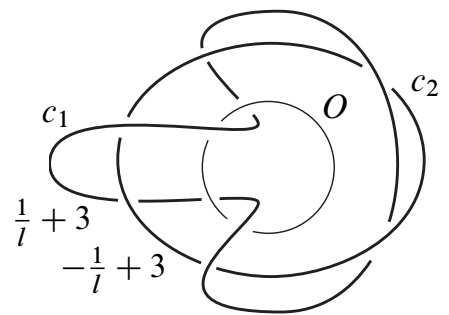

Figure 7: $c_{1}$ and $c_{2}$ become fibers in a Seifert fibration of $O(0)$.

Example 3.5 Let us consider the three-component link $O \cup c_{1} \cup c_{2}$ depicted in Figure 7. It is shown in [13, Lemma 9.26] that $c_{1}$ and $c_{2}$ become fibers in a Seifert fibration of $O(0)$. Let $A$ be an annulus in $S^{3}$ cobounded by $c_{1}$ and $c_{2}$. Performing a $(-l)-$ annulus twist along $A$ (equivalently performing $(1 / l+3)$ - and $(-1 / l+3)$-surgeries on $c_{1}$ and $c_{2}$, respectively), we obtain a knot $K_{l}$ given by Eudave-Muñoz [15]. Then, as he shows in that paper, $\left(K_{l}, 12 l^{2}-4 l\right)$ is a Seifert surgery such that $K_{l}\left(12 l^{2}-4 l\right)$ is a Seifert fiber space over $\mathbb{R} P^{2}$ with at most two exceptional fibers $c_{1}$ and $c_{2}$ of indices $|l|$ and $|-3 l+1|$ for $l \neq 0$. Here we use the same symbol $c_{i}$ to denote the image of $c_{i}$ after the $(-l)$-annulus twist along $A$. Let $c$ be one of $c_{1}$ or $c_{2}$. Then $c$ is a seiferter for $\left(K_{l}, 12 l^{2}-4 l\right)$. Theorem 1.5 shows that a knot $K_{l, n}$ obtained from $K_{l}$ by an $n$-twist along $c$ is an L-space knot for all integers $n$.

\section{L-space surgeries and twisting along seiferters, II: Degenerate case}

In this section we will prove Theorem 1.6.

Proof of Theorem 1.6 Since $K(m)$ has a degenerate Seifert fibration, it is a lens space or a connected sum of two lens spaces; see [13, Proposition 2.8].

(1) Suppose that $K(m)$ is a lens space with degenerate Seifert fibration. Then there are at most two degenerate fibers in $K(m)$ [13, Proposition 2.8]. Assume that there are exactly two degenerate fibers. Then $(K, m)=(O, 0)$, and the exterior of these two degenerate fibers is $S^{1} \times S^{1} \times[0,1]$. If $c$ is a nondegenerate fiber, then $K_{n}\left(m_{n}\right)$ has $S^{2} \times S^{1}$ as a connected summand for all integers $n$, and thus $\left(K_{n}, m_{n}\right)=(O, 0)$ for all integers $n$ [17, Theorem 8.1]. This contradicts [13, Theorem 5.1]. If $c$ is one of the degenerate fibers, then $\left(K_{n}, m_{n}\right)$ is a lens space, which is $S^{2} \times S^{1}$ only when $\left(K_{n}, m_{n}\right)=(O, 0)=\left(K_{0}, m_{0}\right)$, ie $n=0$, [13, Theorem 5.1]. Thus $\left(K_{n}, m_{n}\right)$ is an L-space surgery except when $n=0$. 
Suppose that $K(m)$ has exactly one degenerate fiber $t_{d}$. There are two cases to consider: $K(m)$ - int $N\left(t_{d}\right)$ is a fibered solid torus, or it has a nondegenerate Seifert fibration over the Möbius band with no exceptional fiber [13, Proposition 2.8]. In either case, a meridian of $t_{d}$ is identified with a regular fiber on $\partial\left(K(m)-\operatorname{int} N\left(t_{d}\right)\right)$.

Assume that $K(m)-$ int $N\left(t_{d}\right)$ is a fibered solid torus. Suppose that $c$ is a nondegenerate fiber. If $c$ is a core of the solid torus, then $K(m)-$ int $N(c)$ is a solid torus and $K_{n}\left(m_{n}\right)$ is a lens space. Hence $\left(K_{n}, m_{n}\right)$ is an L-space surgery except when $K_{n}\left(m_{n}\right) \cong S^{2} \times S^{1}$, ie when $\left(K_{n}, m_{n}\right)=(O, 0)$. By [13, Theorem 5.1], there is at most one such integer $n$. If $c$ is not a core in the fibered solid torus $K(m)-\operatorname{int} N\left(t_{d}\right)$, then $K_{n}\left(m_{n}\right)$ is a lens space ( $¥ S^{2} \times S^{1}$ ), a connected sum of two lens spaces, or a connected sum of $S^{2} \times S^{1}$ and a lens space $\left(\not S^{3}, S^{2} \times S^{1}\right)$. The last case cannot happen for homological reasons, and hence $\left(K_{n}, m_{n}\right)$ is an L-space surgery. If $c$ is the degenerate fiber $t_{d}$, then $K_{n}\left(m_{n}\right)$ is a lens space, and except for possibly an integer $n_{0}$ with $\left(K_{n_{0}}, m_{n_{0}}\right)=(O, 0)$, we have that $\left(K_{n}, m_{n}\right)$ is an L-space surgery.

Next consider the case where $K(m)-$ int $N\left(t_{d}\right)$ has a nondegenerate Seifert fibration over the Möbius band. Then $(K, m)=(O, 0)$; see [13, Proposition 2.8]. If $c$ is a nondegenerate fiber, $K_{n}\left(m_{n}\right)$ has $S^{2} \times S^{1}$ as a connected summand for all integers $n$. This implies that $\left(K_{n}, m_{n}\right)=(O, 0)$ for all $n$ by [17, Theorem 8.1], contradicting [13, Theorem 5.1]. Thus $c$ is a degenerate fiber, and $K_{n}\left(m_{n}\right)(n \neq 0)$ is a Seifert fiber space over $\mathbb{R} P^{2}$ with at most one exceptional fiber, which has finite fundamental group. Hence if $n$ is any nonzero integer, then $\left(K_{n}, m_{n}\right)$ is an L-space [47, Proposition 2.3]. It follows that if $c$ is a fiber in a degenerate Seifert fibration of a lens space $K(m)$, then $(K, m)$ is an L-space surgery except for at most one integer $n$.

(2) Next suppose that $K(m)$ is a connected sum of two lens spaces. It follows from [13, Proposition 2.8] that $K(m)$ has exactly one degenerate fiber $t_{d}$, and $K(m)-\operatorname{int} N\left(t_{d}\right)$ is a Seifert fiber space over the disk with two exceptional fibers. Note that a meridian of $t_{d}$ is identified with a regular fiber on $\partial\left(K(m)-\right.$ int $\left.N\left(t_{d}\right)\right)$. We divide into two cases: $c$ is a nondegenerate fiber or a degenerate fiber.

(i) First assume that $c$ is a nondegenerate fiber. By [13, Corollary 3.21 (1)], $c$ is not a regular fiber. Hence $c$ is an exceptional fiber, and $K_{n}\left(m_{n}\right)$ is a lens space ( $¥ S^{2} \times S^{1}$ ), a connected sum of two lens spaces, or a connected sum of $S^{2} \times S^{1}$ and a lens space $\left(\not S^{3}, S^{2} \times S^{1}\right)$. The last case cannot happen for homological reasons. Hence $\left(K_{n}, m_{n}\right)$ is an L-space surgery for any integer $n$.

(ii) Now assume that $c$ is a degenerate fiber, ie $c=t_{d}$. As in the proof of Theorem 1.4, let $E$ be $K(m)-$ int $N(c)$ with a fibered tubular neighborhood of the union of two exceptional fibers $t_{1}$ and $t_{2}$ and one regular fiber $t_{0}$ removed. Then $E$ is a product circle bundle over the fourth-punctured sphere. Take a cross section of $E$ such that $K(m)$ 
has a Seifert invariant $S^{2}\left(b, r_{1}, r_{2}, 1 / 0\right)$, where the Seifert invariant of $t_{0}$ is $b \in \mathbb{Z}$, that of $t_{i}$ is $0<r_{i}<1$ for $i=1,2$, and that of $c$ is $1 / 0$. We may assume that $r_{1} \leq r_{2}$. Let $s$ be the boundary curve on $\partial N(c)$ of the cross section so that $[s] \cdot[t]=1$ for a regular fiber $t \subset \partial N(c)$. Then $[\mu]=[t] \in H_{1}(\partial N(c))$ and $[\lambda]=-[s]-\beta[t] \in H_{1}(\partial N(c))$ for some integer $\beta$, ie we have

$$
\left(\begin{array}{l}
{[\mu]} \\
{[\lambda]}
\end{array}\right)=\left(\begin{array}{rr}
0 & 1 \\
-1 & -\beta
\end{array}\right)\left(\begin{array}{l}
{[s]} \\
{[t]}
\end{array}\right) .
$$

Let $c_{n}$ be the image of $c$ after an $n$-twist along $c$. Then the argument in the proof of Theorem 1.4 shows that a preferred meridian-longitude pair $\left(\mu_{n}, \lambda_{n}\right)$ of $\partial N\left(c_{n}\right)$ has the expression

$$
\left(\begin{array}{l}
{\left[\mu_{n}\right]} \\
{\left[\lambda_{n}\right]}
\end{array}\right)=\left(\begin{array}{cc}
n & n \beta+1 \\
-1 & -\beta
\end{array}\right)\left(\begin{array}{l}
{[s]} \\
{[t]}
\end{array}\right) .
$$

Thus $K_{n}\left(m_{n}\right)=S^{2}\left(b, r_{1}, r_{2},(n \beta+1) / n\right)=S^{2}\left(b+\beta, r_{1}, r_{2},(n \beta+1) / n-\beta\right)=$ $S^{2}\left(b+\beta, r_{1}, r_{2}, 1 / n\right)$ for a nonzero integer $n$.

Claim 4.1 $K_{n}\left(m_{n}\right)$ is an $L$-space for $n=0, \pm 1$.

Proof of claim Recall that $K_{0}\left(m_{0}\right)=K(m)$ is a connected sum of two lens spaces $L_{1}$ and $L_{2}$ such that $H_{1}\left(L_{1}\right) \cong \mathbb{Z}_{\alpha_{1}}$ and $H_{1}\left(L_{2}\right) \cong \mathbb{Z}_{\alpha_{2}}$, where $r_{i}=\beta_{i} / \alpha_{i}$. Thus $K_{0}\left(m_{0}\right)$ is an L-space. Since $K_{-1}\left(m_{-1}\right)$ and $K_{1}\left(m_{1}\right)$ are lens spaces, it remains to show that they are not $S^{2} \times S^{1}$. Assume for a contradiction that $K_{1}\left(m_{1}\right)$ or $K_{-1}\left(m_{-1}\right)$ is $S^{2} \times S^{1}$. Then Claim 2.5 shows that $r_{1}+r_{2}=1$; hence $r_{2}=\beta_{2} / \alpha_{2}=\left(\alpha_{1}-\beta_{1}\right) / \alpha_{1}$. Thus $\alpha_{1}=\alpha_{2}$, and $H_{1}\left(K_{0}\left(m_{0}\right)\right) \cong \mathbb{Z}_{\alpha_{1}} \oplus \mathbb{Z}_{\alpha_{2}}$ is not cyclic, a contradiction. Hence neither $K_{1}\left(m_{1}\right)$ nor $K_{-1}\left(m_{-1}\right)$ is $S^{2} \times S^{1}$ and they are L-spaces.

(1) If $b+\beta \leq-3$ or $b+\beta \geq 1$, then Proposition 2.4(1) shows that $K_{n}\left(m_{n}\right)=$ $S^{2}\left(b+\beta, r_{1}, r_{2}, 1 / n\right)$ is an L-space if $-1 \leq 1 / n \leq 1$, ie $n \leq-1$ or $n \geq 1$. See Figure $8\left(\right.$ left). Since $K_{0}\left(m_{0}\right)$ is also an L-space (Claim 4.1), $K_{n}\left(m_{n}\right)$ is an L-space for any integer $n$.

(2) If $b+\beta=-2$, Proposition 2.4(2) shows that there is an $\varepsilon>0$ such that $K_{n}\left(m_{n}\right)=$ $S^{2}\left(b+\beta, r_{1}, r_{2}, 1 / n\right)$ is an L-space if $-1 \leq 1 / n \leq \varepsilon$. Hence $K_{n}\left(m_{n}\right)$ is an L-space if $n \leq-1$ or $n \geq 1 / \varepsilon$. See Figure 8(middle). This, together with Claim 4.1, shows that $K_{n}\left(m_{n}\right)$ is an L-space if $n \leq 1$ or $n \geq 1 / \varepsilon$.

(3) Suppose that $b+\beta=-1$. Then Proposition 2.4(3) shows that if $r_{1}+r_{2} \geq 1$ (resp. $\left.r_{1}+r_{2} \leq 1\right), K_{n}\left(m_{n}\right)=S^{2}\left(b+\beta, r_{1}, r_{2}, 1 / n\right)$ is an L-space for any integer $n$ satisfying $0<1 / n \leq 1$, ie $n \geq 1$ (resp. $-1 \leq 1 / n<0$, ie $n \leq-1$ ). See Figure 8 (left). 

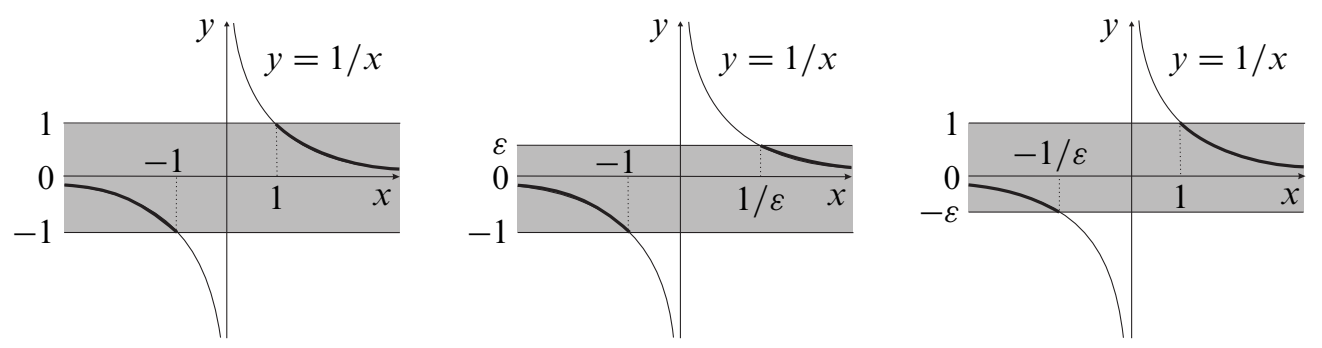

Figure 8: Left: $-1 \leq 1 / n \leq 1$ if $n \leq-1$ or $n \geq 1$. Middle: $-1 \leq 1 / n \leq \varepsilon$ if $n \leq-1$ or $n \geq 1 / \varepsilon$. Right: $-\varepsilon \leq 1 / n \leq 1$ if $n \leq-1 / \varepsilon$ or $n \geq 1$.

Combining this with Claim 4.1, we see that $K_{n}\left(m_{n}\right)$ is an L-space for any $n \geq-1$ (resp. $n \leq 1$ ).

(4) If $b+\beta=0$, then Proposition 2.4(4) shows that there is an $\varepsilon>0$ such that $K_{n}\left(m_{n}\right)=S^{2}\left(b+\beta, r_{1}, r_{2}, 1 / n\right)$ is an L-space if $-\varepsilon \leq 1 / n \leq 1$. Hence $K_{n}\left(m_{n}\right)$ is an L-space if $n \geq 1$ or $n \leq-1 / \varepsilon$. See Figure 8(right). This, together with Claim 4.1, shows that $K_{n}\left(m_{n}\right)$ is an L-space if $n \geq-1$ or $n \leq-1 / \varepsilon$.

This completes the proof of Theorem 1.6.

As shown by Greene in [23, Theorem 1.5], if $K(m)$ is a connected sum of lens spaces, then $K$ is a torus knot or a cable of a torus knot. More precisely, $(K, m)=\left(T_{p, q}, p q\right)$ or $\left(C_{p, q}\left(T_{r, s}\right), p q\right)$, where $p=q r s \pm 1$. Note that $T_{p, q}(p q)=L(p, q) \# L(q, p)$ and $C_{p, q}\left(T_{r, s}\right)(p q)=L\left(p, q s^{2}\right) \# L(q, \pm 1)$.

Let us continue to prove Theorem 1.7, which is a refinement of Theorem 1.6(2).

Proof of Theorem 1.7 Henceforth, $(K, m)$ is either $\left(T_{p, q}, p q\right)$ or $\left(C_{p, q}\left(T_{r, s}\right), p q\right)$, where $p, q \geq 2$ and $p=q r s \pm 1$. If $c$ becomes a nondegenerate fiber in $K(m)$, then as shown in the proof of Theorem 1.6, $K_{n}$ is an L-space knot for any integer $n$. So we assume that $c$ becomes a degenerate fiber in $K(m)$. Recall from [13, Theorem 3.19 (3)] that the linking number $l$ between $c$ and $K$ is not zero. Recall also that $K_{n}\left(m_{n}\right)$ is expressed as $S^{2}\left(b+\beta, r_{1}, r_{2}, 1 / n\right)=S^{2}\left(b+\beta, \beta_{1} / \alpha_{1}, \beta_{2} / \alpha_{2}, 1 / n\right)$, where $\alpha_{i} \geq 2$ and $0<r_{i}=\beta_{i} / \alpha_{i}<1$. See the proof of Theorem 1.6. Note that $\left\{\alpha_{1}, \alpha_{2}\right\}=\{p, q\}$, and $\alpha_{1} \alpha_{2}=p q \geq 6$.

Claim 4.2 $b+\beta \neq-2$.

Proof of claim Assume for a contradiction that $b+\beta=-2$. Then $K_{1}\left(m_{1}\right)=$ $S^{2}\left(-2, \beta_{1} / \alpha_{1}, \beta_{2} / \alpha_{2}, 1\right)=S^{2}\left(-1, \beta_{1} / \alpha_{1}, \beta_{2} / \alpha_{2}\right)$. Therefore, $\left|H_{1}\left(K_{1}\left(m_{1}\right)\right)\right|=$ $\left|-\alpha_{1} \alpha_{2}+\alpha_{1} \beta_{2}+\alpha_{2} \beta_{1}\right|$, which equals $p q+l^{2}=\alpha_{1} \alpha_{2}+l^{2}$. Since $\alpha_{1} \alpha_{2}+l^{2}>\alpha_{1} \alpha_{2}$, 
we have $\left|-\alpha_{1} \alpha_{2}+\alpha_{1} \beta_{2}+\alpha_{2} \beta_{1}\right|>\alpha_{1} \alpha_{2}$. This then implies $\beta_{1} / \alpha_{1}+\beta_{2} / \alpha_{2}>2$ or $\beta_{1} / \alpha_{1}+\beta_{2} / \alpha_{2}<0$. Neither case can happen, because $0<\beta_{i} / \alpha_{i}<1$. Thus $b+\beta \neq-2$.

Claim 4.3 If $b+\beta=-1$, then $\beta_{1} / \alpha_{1}+\beta_{2} / \alpha_{2}>1$.

Proof of claim If $b+\beta=-1$, then we have $K_{1}\left(m_{1}\right)=S^{2}\left(-1, \beta_{1} / \alpha_{1}, \beta_{2} / \alpha_{2}, 1\right)=$ $S^{2}\left(\beta_{1} / \alpha_{1}, \beta_{2} / \alpha_{2}\right)$. Thus $\left|H_{1}\left(K_{1}\left(m_{1}\right)\right)\right|=\alpha_{1} \beta_{2}+\alpha_{2} \beta_{1}$, which is equal to $p q+l^{2}=$ $\alpha_{1} \alpha_{2}+l^{2}$. Since $\alpha_{1} \alpha_{2}+l^{2}>\alpha_{1} \alpha_{2}$, we have $\alpha_{1} \beta_{2}+\alpha_{2} \beta_{1}>\alpha_{1} \alpha_{2}$. This shows $\beta_{1} / \alpha_{1}+\beta_{2} / \alpha_{2}>1$.

Claims 4.2 and 4.3, together with the argument in the proof of Theorem 1.6, prove that $K_{n}$ is an L-space knot for any $n \geq-1$.

Now let us prove that $K_{n}$ is an L-space knot for all integers $n$ under the assumption that $l^{2} \geq 2 p q$.

Claim 4.4 If $l^{2} \geq 2 p q$, then $b+\beta \neq-1$.

Proof of claim Assume that $l^{2} \geq 2 p q$, and suppose that $b+\beta=-1$ for a contradiction. Then $K_{-1}\left(m_{-1}\right)=S^{2}\left(-1, \beta_{1} / \alpha_{1}, \beta_{2} / \alpha_{2},-1\right)=S^{2}\left(-2, \beta_{1} / \alpha_{1}, \beta_{2} / \alpha_{2}\right)$, and $\left|H_{1}\left(K_{-1}\left(m_{-1}\right)\right)\right|=\left|-2 \alpha_{1} \alpha_{2}+\alpha_{1} \beta_{2}+\alpha_{2} \beta_{1}\right|$, which equals $\left|p q-l^{2}\right|$. The assumption $l^{2} \geq 2 p q=2 \alpha_{1} \alpha_{2}$ implies that $\left|p q-l^{2}\right|=l^{2}-p q=l^{2}-\alpha_{1} \alpha_{2} \geq \alpha_{1} \alpha_{2}$. Hence $\left|-2 \alpha_{1} \alpha_{2}+\alpha_{1} \beta_{2}+\alpha_{2} \beta_{1}\right|=\left|p q-l^{2}\right| \geq \alpha_{1} \alpha_{2}$. Thus we have $\beta_{1} / \alpha_{1}+\beta_{2} / \alpha_{2} \geq 3$ or $\beta_{1} / \alpha_{1}+\beta_{2} / \alpha_{2} \leq 1$. The former case cannot happen because $0<\beta_{i} / \alpha_{i}<1$, and the latter case contradicts Claim 4.3 which asserts that $\beta_{1} / \alpha_{1}+\beta_{2} / \alpha_{2}>1$. Hence $b+\beta \neq-1$.

Claim 4.5 If $l^{2} \geq 2 p q$, then $b+\beta \neq 0$.

Proof of claim Suppose for a contradiction that $b+\beta=0$. Then

$$
K_{-1}\left(m_{-1}\right)=S^{2}\left(0, \beta_{1} / \alpha_{1}, \beta_{2} / \alpha_{2},-1\right)=S^{2}\left(-1, \beta_{1} / \alpha_{1}, \beta_{2} / \alpha_{2}\right),
$$

and thus $\left|H_{1}\left(K_{-1}\left(m_{-1}\right)\right)\right|=\left|-\alpha_{1} \alpha_{2}+\alpha_{1} \beta_{2}+\alpha_{2} \beta_{1}\right|$, which equals $\left|p q-l^{2}\right|$. Since $l^{2} \geq 2 p q=2 \alpha_{1} \alpha_{2}$, we have $\left|p q-l^{2}\right|=l^{2}-p q=l^{2}-\alpha_{1} \alpha_{2} \geq \alpha_{1} \alpha_{2}$. Therefore $\left|-\alpha_{1} \alpha_{2}+\alpha_{1} \beta_{2}+\alpha_{2} \beta_{1}\right|=\left|p q-l^{2}\right| \geq \alpha_{1} \alpha_{2}$. This then implies $\beta_{1} / \alpha_{1}+\beta_{2} / \alpha_{2} \geq 2$ or $\beta_{1} / \alpha_{1}+\beta_{2} / \alpha_{2} \leq 0$. Neither case can happen, because $0<\beta_{i} / \alpha_{i}<1$. Thus $b+\beta \neq 0$. 


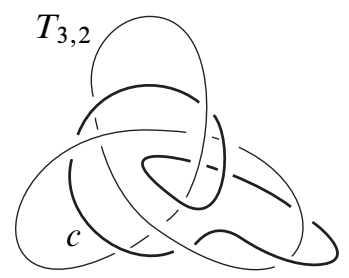

Figure 9: $c$ is a seiferter for $\left(T_{3,2}, 6\right)$.

Under the assumption $l^{2} \geq 2 p q$, Claims $4.2,4.4$ and 4.5 imply that $b+\beta \leq-3$ or $b+\beta \geq 1$. Then the proof of Theorem 1.6 enables us to conclude that $K_{n}$ is an L-space knot for all integers $n$. This completes the proof of Theorem 1.7.

Example 4.6 Let $K$ be a torus knot $T_{3,2}$ and $c$ an unknotted circle as depicted in Figure 9; the linking number between $c$ and $T_{3,2}$ is 5 . Then $c$ coincides with $c_{3,2}^{+}$in Section 5, and it is a seiferter for $\left(T_{3,2}, 6\right)$. Let $K_{n}$ be a knot obtained from $T_{3,2}$ by an $n$-twist along $c$. Since $5^{2}>2 \cdot 3 \cdot 2=12$, following Theorem $1.7 K_{n}$ is an L-space knot for all integers $n$.

Example 4.7 below gives an example of a seiferter for $(K, m)$, where $K$ is a cable of a torus knot and $K(m)$ is a connected sum of two lens spaces.

Example 4.7 Let $k$ be a Berge knot Spor $\boldsymbol{a}[p](p>1)$. Then $k\left(22 p^{2}+9 p+1\right)$ is a lens space, and [12, Proposition 8.1 and Table 9] show that $\left(k, 22 p^{2}+9 p+1\right)$ has a seiferter $c$ such that the linking number between $c$ and $k$ is $4 p+1$ and a $(-1)$-twist along $c$ converts $\left(k, 22 p^{2}+9 p+1\right)$ into $\left(C_{6 p+1, p}\left(T_{3,2}\right), p(6 p+1)\right)$. Since $p>1, C_{6 p+1, p}\left(T_{3,2}\right)$ is a nontrivial cable of $T_{3,2}$. Thus $c$ is a seiferter for $\left(C_{6 p+1, p}\left(T_{3,2}\right), p(6 p+1)\right)$. Let $K_{n}$ be a knot obtained from $C_{6 p+1, p}\left(T_{3,2}\right)$ by an $n$-twist along $c$ so that $K_{1}=k$. Since $(4 p+1)^{2} \geq 2(6 p+1) p$, Theorem 1.7 shows that $K_{n}$ is an L-space knot for all integers $n$.

Finally, we show that $K_{n}$ is hyperbolic if $|n|>3$. As shown in [12, Figure 41], $K_{n}$ admits a Seifert surgery yielding a small Seifert space which is not a lens space, so we see that $c$ becomes a degenerate fiber in $C_{6 p+1, p}\left(T_{3,2}\right)(p(6 p+1))$ [13, Lemma $\left.5.6(1)\right]$. Hence [13, Corollary 3.21 (3)] shows that the link $C_{6 p+1, p}\left(T_{3,2}\right) \cup c$ is hyperbolic. The result now follows from [13, Proposition 5.11 (3)].

We close this section with the following observation, which shows the nonuniqueness of a degenerate Seifert fibration of a connected sum of two lens spaces.

Let $c$ be a seiferter for $\left(T_{p, q}, p q\right)$ which becomes a degenerate fiber in $T_{p, q}(p q)$. As the simplest example of such a seiferter $c$, take a meridian $c_{\mu}$ of $T_{p, q}$. Then 


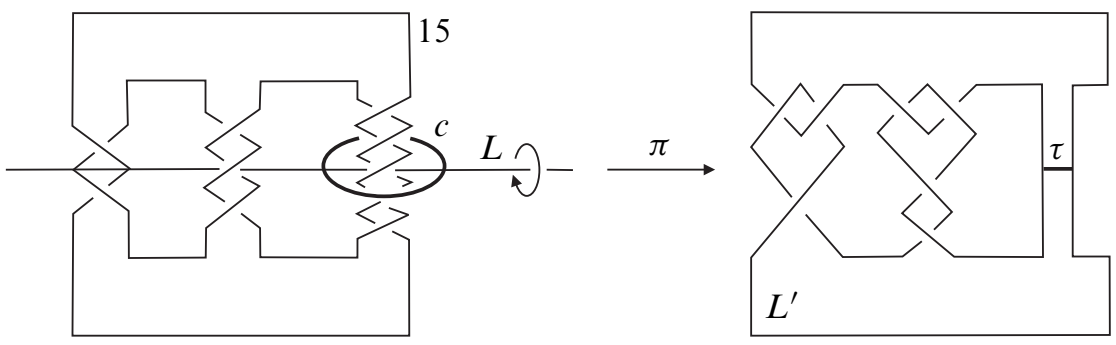

$K=T_{5,3}=P(-2,3,5)$

Figure 10: $T_{5,3}(15)$ is the two-fold branched cover of $S^{3}$ branched along $L^{\prime}$.

$c_{\mu}$ is isotopic to the core of the filled solid torus (ie the dual knot of $T_{p, q}$ ) in $T_{p, q}(p q)$, which is a degenerate fiber. Hence $c_{\mu}$ is a seiferter for $\left(T_{p, q}, p q\right)$ which becomes a degenerate fiber in $T_{p, q}(p q)$, and $T_{p, q}-$ int $N\left(c_{\mu}\right)$ is homeomorphic to $S^{3}-$ int $N\left(T_{p, q}\right)$. However, in general, $T_{p, q}(p q)-$ int $N(c)$ is not necessarily homeomorphic to $S^{3}-$ int $N\left(T_{p, q}\right)$.

Example 4.8 Let us take an unknotted circle $c$ as in Figure 10. Then $c$ is a seiferter for $\left(T_{5,3}, 15\right)$ which becomes a degenerate fiber in $T_{5,3}(15)$, but $T_{5,3}(15)-\operatorname{int} N(c)$ is not homeomorphic to $S^{3}-\operatorname{int} N\left(T_{5,3}\right)$.

Proof As shown in Figure 10, $T_{5,3}(15)$ is the two-fold branched cover of $S^{3}$ branched along $L^{\prime}$, and $c$ is the preimage of an arc $\tau$. Hence $T_{5,3}(15)-\operatorname{int} N(c)$ is a Seifert fiber space $D^{2}(2 / 3,-2 / 5)$. Since $\left|H_{1}\left(S^{2}(2 / 3,-2 / 5, x)\right)\right|=|4+15 x|$ cannot be 1 for any integer $x$, the Seifert fiber space $T_{5,3}(15)-$ int $N(c)$ cannot be embedded in $S^{3}$, and hence it is not homeomorphic to $S^{3}-$ int $N\left(T_{5,3}\right)$. (Note that $c$ coincides with $c_{5,3}^{-}$in Section 5.)

\section{L-space twisted torus knots}

Each torus knot obviously has an unknotted circle $c$ which satisfies the desired property in Question 1.1.

Example 5.1 Embed a torus knot $T_{p, q}$ into a genus-one Heegaard surface of $S^{3}$. Then cores of the Heegaard splitting $s_{p}$ and $s_{q}$ are seiferters for $\left(T_{p, q}, m\right)$ for all integers $m$. We call them basic seiferters for $T_{p, q}$; see Figure 11. An $n$-twist along $s_{p}$ (resp. $s_{q}$ ) converts $T_{p, q}$ into a torus knot $T_{p+n q, q}\left(\right.$ resp. $T_{p, q+n p}$ ), and hence an $n$-twist along a basic seiferter yields an L-space knot for all $n$. 


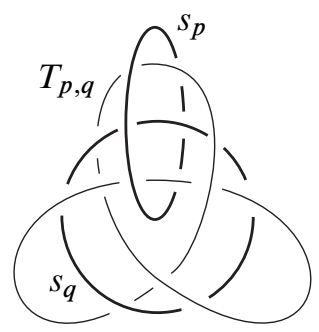

Figure 11: $s_{p}$ and $s_{q}$ are basic seiferters for $\left(T_{p, q}, m\right)$.

Twistings along a basic seiferter keep the property of being L-space knots, but produce only torus knots. In the following, we will give another circle $c$ such that twistings of $T_{p, q}$ along $c$ produce an infinite family of hyperbolic L-space knots.

Definition 5.2 [10] Let $\Sigma$ denote a genus-one Heegaard surface of $S^{3}$. Let $T_{p, q}$ $(p>q \geq 2)$ be a $(p, q)$-torus knot which lies on $\Sigma$. Choose an unknotted circle $c \subset S^{3}-T_{p, q}$ so that it bounds a disk $D$ such that $D \cap \Sigma$ is a single arc intersecting $T_{p, q}$ in $r(2 \leq r \leq p+q)$ points in the same direction. A twisted torus knot $K(p, q ; r, n)$ is a knot obtained from $T_{p, q}$ by adding $n$ full twists along $c$.

Remark 5.3 Twisting $T_{p, q}$ along the basic seiferter $s_{p}$ (resp. $s_{q}$ ) $n$ times, we obtain the twisted torus knot $K(p, q ; q, n)$ (resp. $K(p, q ; p, n))$, which is a torus knot $T_{p+n q, q}$ (resp. $T_{p, q+n p}$ ), and hence an L-space knot.

In [53], Vafaee studied twisted torus knots from a viewpoint of knot Floer homology and showed that twisted torus knots $K(p, k p \pm 1 ; r, n)$, where $p \geq 2, k \geq 1, n>0$ and $0<r<p$, are L-space knots if and only if either $r=p-1$ or $r \in\{2, p-2\}$ and $n=1$. We will give yet more twisted torus knots which are L-space knots by combining seiferter technology and Theorem 1.7.

Proof of Theorem 1.8 In the following, let $\Sigma$ be a genus-one Heegaard surface of $S^{3}$, which bounds solid tori $V_{1}$ and $V_{2}$.

$\boldsymbol{K}(\boldsymbol{p}, \boldsymbol{q} ; \boldsymbol{p}+\boldsymbol{q}, \boldsymbol{n})(\boldsymbol{p}>\boldsymbol{q} \geq \mathbf{2}) \quad$ Given any torus knot $T_{p, q}(p>q \geq 2)$ on $\Sigma$, let us take an unknotted circle $c_{p . q}^{+}$in $S^{3}-T_{p, q}$ as depicted in Figure 12(left); the linking number between $c_{p, q}^{+}$and $T_{p, q}$ is $p+q$.

Let $V$ be the solid torus $S^{3}-$ int $N\left(c_{p, q}^{+}\right)$, which contains $T_{p, q}$ in its interior. Then [36, Lemma 9.1] shows that $V(K ; p q)=T_{p, q}(p q)-$ int $N\left(c_{p, q}^{+}\right)$is a Seifert fiber space over the disk with two exceptional fibers of indices $p, q$, and a meridian of $N\left(c_{p, q}^{+}\right)$coincides with a regular fiber on $\partial V(K ; p q)$. Hence $c_{p, q}^{+}$is a degenerate fiber in $T_{p, q}(p q)$, and thus it is a seiferter for $\left(T_{p, q}, p q\right)$. Let $D$ be a disk bounded by $c_{p, q}^{+}$. 

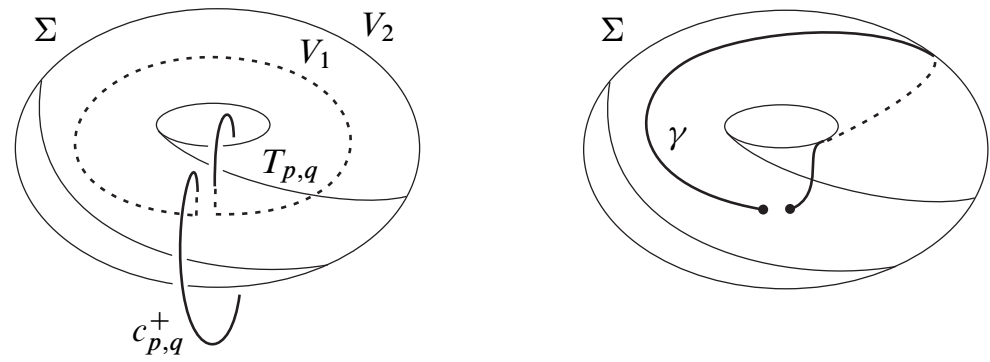

Figure 12: $c_{p, q}^{+}$is a seiferter for $\left(T_{p, q}, p q\right)$.
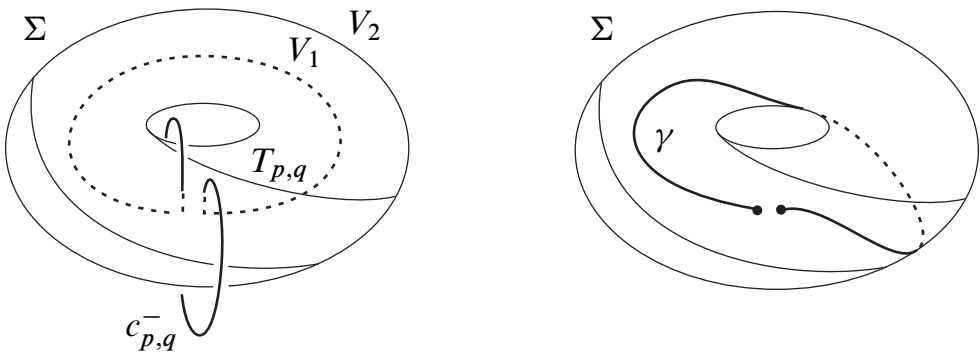

Figure 13: $c_{p, q}^{-}$is a seiferter for $\left(T_{p, q}, p q\right)$.

Since the arc $c_{p, q}^{+} \cap V_{i}$ is isotoped in $V_{i}$ to an arc $\gamma \subset \Sigma$ depicted in Figure 12(right) leaving its endpoints fixed, the disk $D$ can be isotoped so that $D \cap \Sigma=\gamma$, which intersects $T_{p, q}$ in $p+q$ points in the same direction. Thus an $n$-twist along $c_{p, q}^{+}$ converts $T_{p, q}$ into the twisted torus knot $K(p, q ; p+q, n)$. Since $c_{p, q}^{+}$is a seiferter for $\left(T_{p, q}, p q\right)$ and $(p+q)^{2}=p^{2}+q^{2}+2 p q>2 p q$, we can apply Theorem 1.7 to conclude that $T(p, q, p+q, n)$ is an L-space knot for all integers $n$.

We now show that $T(p, q, p+q, n)$ is hyperbolic if $|n|>3$. By a linking number consideration, we see that $c_{p, q}^{+}$is not a basic seiferter. Then [13, Corollary 3.21 (3)] (see also [36, Claim 9.2]) shows that $T_{p, q} \cup c_{p . q}^{+}$is a hyperbolic link. Thus [13, Proposition 5.11 (2)] shows that $K(p, q ; p+q, n)$ is a hyperbolic knot if $|n|>3$.

$\boldsymbol{K}(\boldsymbol{p}, \boldsymbol{q} ; \boldsymbol{p}-\boldsymbol{q}, \boldsymbol{n})(\boldsymbol{p}>\boldsymbol{q} \geq \mathbf{2}) \quad$ Suppose that $p-q \neq 1$. Then let us take $c_{p, q}^{-}$as in Figure 13(left) instead of $c_{p, q}^{+}$; the linking number between $c_{p, q}^{-}$and $T_{p, q}$ is $p-q$. It follows from [13, Remark 4.7] that $c_{p, q}^{-}$is also a seiferter for $\left(T_{p, q}, p q\right)$, and the link $T_{p, q} \cup c_{p, q}^{-}$is hyperbolic. Note that if $p-q=1$, then $c_{p, q}^{-}$is a meridian of $T_{p, q}$. As above, we see that each arc $c_{p, q}^{-} \cap V_{i}$ is isotoped in $V_{i}$ to an arc $\gamma \subset \Sigma$ depicted in Figure 13(right) leaving its endpoints fixed. So a disk $D$ bounded by $c_{p, q}^{-}$can be isotoped so that $D \cap \Sigma=\gamma$, which intersects $T_{p, q}$ in $p-q$ points in the same direction. Thus an $n$-twist along $c_{p, q}^{-}$converts $T_{p, q}$ into the twisted torus knot $K(p, q ; p-q, n)$. Since $c_{p, q}^{-}$is a seiferter for $\left(T_{p, q}, p q\right)$, Theorem 1.7 shows that $T(p, q, p-q, n)$ is an 


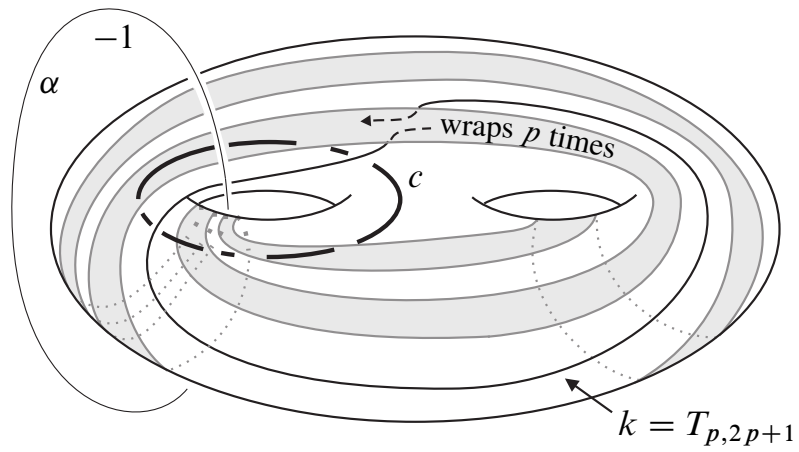

Figure 14: A surgery description of $T_{3 p+1,2 p+1}$ and a seiferter $c$.

L-space knot for any $n \geq-1$. Following [13, Proposition 5.11 (2)], $T(p, q, p-q, n)$ is a hyperbolic knot if $|n|>3$.

$\boldsymbol{K}(\mathbf{3} \boldsymbol{p}+\mathbf{1}, \mathbf{2} \boldsymbol{p}+\mathbf{1} ; \mathbf{4} \boldsymbol{p}+\mathbf{1}, \boldsymbol{n})(\boldsymbol{p}>\mathbf{0})$ Let $k$ be a torus knot $T_{p, 2 p+1}$ on a genustwo Heegaard surface, with unknotted circles $\alpha$ and $c$ as shown in Figure 14. Applying a 1 -twist along $\alpha$, we obtain a torus knot $T_{3 p+1,2 p+1}$. We continue to use the same symbol $c$ to denote the image of $c$ after a 1 -twist along $\alpha$; the linking number between $c$ and $T_{3 p+1,2 p+1}$ is $4 p+1$. Note that a 1 -twist along $c$ converts $T_{3 p+1,2 p+1}$ into a Berge knot Spor $\boldsymbol{b}[p]$ as shown in [12, Subsection 8.2]. Following [12, Lemma 8.4], $c$ is a seiferter for a lens space surgery

$$
\left(\operatorname{Spor} \boldsymbol{b}[p], 22 p^{2}+13 p+2\right)=\left(\operatorname{Spor} \boldsymbol{b}[p],(3 p+1)(2 p+1)+(4 p+1)^{2}\right) .
$$

Thus $c$ is also a seiferter for $\left(T_{3 p+1,2 p+1},(3 p+1)(2 p+1)\right)$. Let $D$ be a disk bounded by $c$. Then $T_{3 p+1,2 p+1} \cup D$ can be isotoped so that $T_{3 p+1,2 p+1}$ lies on $\Sigma$, and $D \cap \Sigma$ consists of a single arc, which intersects $T_{3 p+1,2 p+1}$ in $4 p+1$ points in the same direction. Thus an $n$-twist along $c$ converts $T_{3 p+1,2 p+1}$ into a twisted torus knot $K(3 p+1,2 p+1 ; 4 p+1, n)$. Since $c$ is a seiferter for $\left(T_{3 p+1,2 p+1},(3 p+1)(2 p+1)\right)$ and $(4 p+1)^{2}>2(3 p+1)(2 p+1)$, Theorem 1.7 shows that $K(3 p+1,2 p+1 ; 4 p+1, n)$ is an L-space knot for all integers $n$.

Let us observe that $K(3 p+1,2 p+1 ; 4 p+1, n)$ is a hyperbolic knot if $|n|>3$. But [12, Figure 44] shows that an $n$-twist converts $\left(T_{3 p+1,2 p+1},(3 p+1)(2 p+1)\right)$ into a Seifert surgery which is not a lens space surgery if $|n| \geq 2$. Hence $c$ becomes a degenerate fiber in $T_{3 p+1,2 p+1}((3 p+1)(2 p+1))$ by [13, Lemma $5.6(1)$ ], and [13, Corollary 3.21 (3)] shows that the link $T_{3 p+1,2 p+1} \cup c$ is hyperbolic. The result now follows from [13, Proposition 5.11 (2)].

$\boldsymbol{K}(\mathbf{3 p}+\mathbf{2}, \mathbf{2} \boldsymbol{p}+\mathbf{1} ; \mathbf{4} \boldsymbol{p}+\mathbf{3}, \boldsymbol{n})(\boldsymbol{p}>\mathbf{0})$ As above, we follow the argument in [12, Subsection 8.3], but we need to take the mirror image at the end. Take a torus knot 


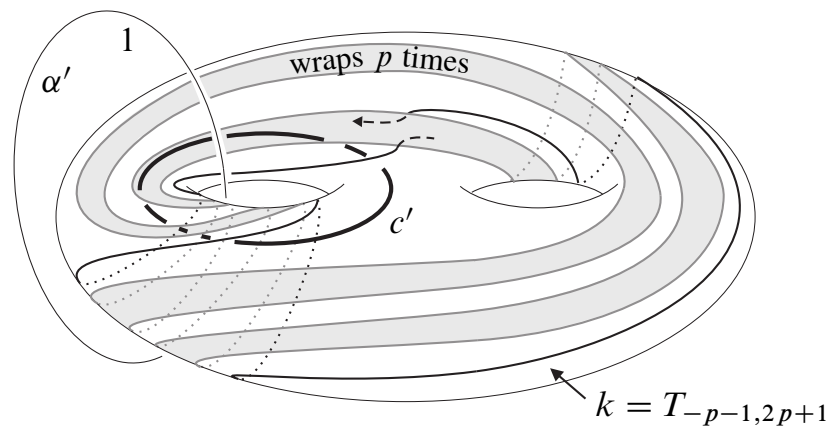

Figure 15: A surgery description of $T_{-3 p-2,2 p+1}$ and a seiferter $c^{\prime}$.

$k=T_{-p-1,2 p+1}$ on a genus-two Heegaard surface of $S^{3}$ and unknotted circles $\alpha^{\prime}$ and $c^{\prime}$ as shown in Figure 15. Then a (-1)-twist along $\alpha^{\prime}$ converts $T_{-p-1,2 p+1}$ into $T_{-3 p-2,2 p+1}$. As above, we denote the image of $c^{\prime}$ after a $(-1)$-twist along $\alpha^{\prime}$ by the same symbol $c^{\prime}$; the linking number between $c^{\prime}$ and $T_{-3 p-2,2 p+1}$ is $4 p+3$. Note that a $(-1)$-twist along $c^{\prime}$ converts $T_{-3 p-2,2 p+1}$ into a Berge knot Spor $c[p]$ as shown in [12, Subsection 8.3]. Then [12, Lemma 8.6] shows that $c^{\prime}$ is a seiferter for a lens space surgery

$$
\left(\operatorname{Spor} c[p],-22 p^{2}-31 p-11\right)=\left(\operatorname{Spor} c[p],(-3 p-2)(2 p+1)-(4 p+3)^{2}\right) .
$$

Thus $c^{\prime}$ is also a seiferter for $\left(T_{-3 p-2,2 p+1},(-3 p-2)(2 p+1)\right)$. Let $D^{\prime}$ be a disk bounded by $c^{\prime}$. Then $T_{-3 p-2,2 p+1} \cup D^{\prime}$ can be isotoped so that $T_{-3 p-2,2 p+1}$ lies on $\Sigma$, and $D^{\prime} \cap \Sigma$ consists of a single arc, which intersects $T_{-3 p-2,2 p+1}$ in $4 p+3$ points in the same direction. Now, taking the mirror image of $T_{-3 p-2,2 p+1} \cup D^{\prime}$, we obtain $T_{3 p+2,2 p+1} \cup D$ with $\partial D=c$; we see $D \cap \Sigma$ consists of a single arc, and $D$ intersects $T_{3 p+2,2 p+1}$ in $4 p+3$ points in the same direction. Then $c$ is a seiferter for $\left(T_{3 p+2,2 p+1},(3 p+2)(2 p+1)\right)$. Since $(4 p+3)^{2}>2(3 p+2)(2 p+1)$, Theorem 1.7 shows that $K(3 p+2,2 p+1 ; 4 p+3, n)$ is an L-space knot for all integers $n$.

Let us now show that $K(3 p+2,2 p+1 ; 4 p+3, n)$ is hyperbolic if $|n|>3$. Figure 47 in [12], together with [13, Lemma $5.6(1)]$, shows that $c^{\prime}$ becomes a degenerate fiber in $T_{-3 p-2,2 p+1}((-3 p-2)(2 p+1))$, and so $c$ becomes a degenerate fiber in $T_{3 p+2,2 p+1}((3 p+2)(2 p+1))$. Apply the same argument as above to obtain the desired result.

$\boldsymbol{K}(\mathbf{2} \boldsymbol{p}+\mathbf{3}, \mathbf{2} \boldsymbol{p}+\mathbf{1} ; \mathbf{2} \boldsymbol{p}+\mathbf{2}, \boldsymbol{n})(\boldsymbol{p}>\mathbf{0})$ We follow the argument in [12, Section 6]; as above; we will take the mirror image at the end. Take a torus knot $k=T_{-3 p-2,3}$ on a genus-two Heegaard surface of $S^{3}$ and unknotted circles $\alpha^{\prime}$ and $c^{\prime}$ as in Figure 16(left). Then a (-2)-twist along $\alpha^{\prime}$ converts the torus knot $T_{-3 p-2,3}$ into a Berge knot VI $[p]$. Thus [12, Lemma 6.1] shows that $c^{\prime}$, the image of $c^{\prime}$ after the (-2)-twist along $\alpha^{\prime}$, is a 

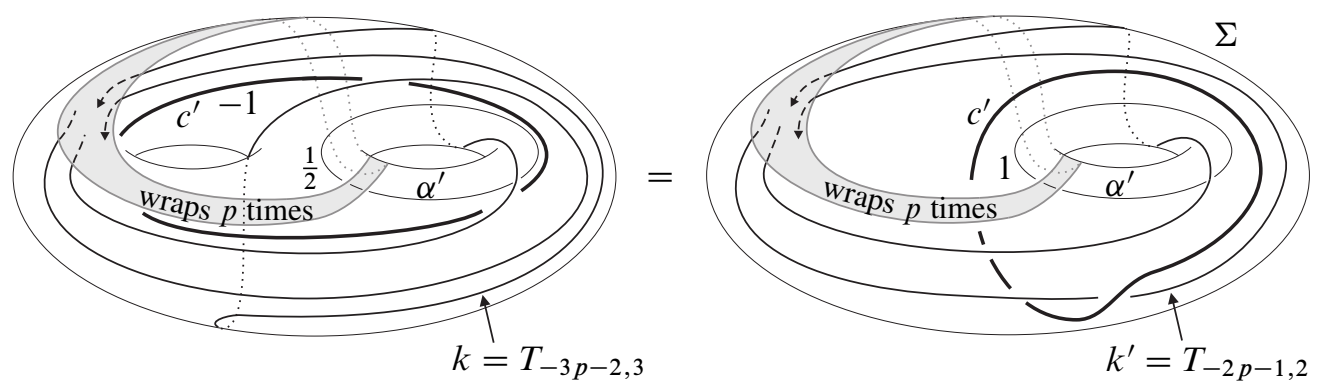

Figure 16: Surgery descriptions of $T_{-2 p-1,2 p+3}$ and a seiferter $c^{\prime}$.

seiferter for a lens space surgery ( VI $\left.[p],-8 p^{2}-16 p-7\right)$; the linking number between $c^{\prime}$ and $\operatorname{VI}[p]$ is $2 p+2$. We now show that a 1 -twist along $c^{\prime}$ (after a $(-2)-$ twist along $\left.\alpha^{\prime}\right)$ converts $\left(\mathrm{VI}[p],-8 p^{2}-16 p-7\right)$ into $\left(T_{-2 p-1,2 p+3},(-2 p-1)(2 p+3)\right)$. Note that $c^{\prime}$ remains a seiferter for $\left(T_{-2 p-1,2 p+3},(-2 p-1)(2 p+3)\right)$. Since the linking number between $c^{\prime}$ and $\operatorname{VI}[p]$ is $2 p+2$, the surgery slope $-8 p^{2}-16 p-7$ becomes $-8 p^{2}-16 p-7+(2 p+2)^{2}=(-2 p-1)(2 p+3)$.

Let us observe that the knot obtained from $\mathrm{VI}[p]$ by a 1 -twist along $c^{\prime}$, which has a surgery description given by Figure 16(left), is $T_{-2 p-1,2 p+3}$. The surgeries described in Figure 16(left) can be realized by the following two successive twistings: a 1twist along an annulus cobounded by $c^{\prime}$ and $\alpha^{\prime}$ (see [13, Definition 2.32]), and a (-1)-twist along $\alpha^{\prime}$. The annulus twist converts $k=T_{-3 p-2,3}$ into $k^{\prime}=T_{-2 p-1,2}$ as shown in Figure 16(right). Then a (-1)-twist along $\alpha^{\prime}$ changes $k^{\prime}=T_{-2 p-1,2}$ into $T_{-2 p-1,2 p+3}$, which lies on the genus-one Heegaard surface $\Sigma$. Let $D^{\prime}$ be a disk bounded by $c^{\prime}$. Then $D^{\prime}$ can be slightly isotoped so that $D^{\prime} \cap \Sigma$ consists of a single arc, which intersects $T_{-2 p-1,2 p+3}$ in $2 p+2$ points in the same direction; see Figure 16(right). Now taking the mirror image of $T_{-2 p-1,2 p+3} \cup D^{\prime}$, we obtain $T_{2 p+1,2 p+3} \cup D$ with $\partial D=c$, and $D \cap \Sigma$ consists of a single arc, which intersects $T_{2 p+1,2 p+3}$ in $2 p+2$ points in the same direction. Then $c$ is a seiferter for

$$
\left(T_{2 p+1,2 p+3},(2 p+1)(2 p+3)\right)=\left(T_{2 p+3,2 p+1},(2 p+3)(2 p+1)\right) .
$$

Theorem 1.7 shows that $K(2 p+3,2 p+1 ; 2 p+2, n)$ is an L-space knot for any integer $n \geq-1$. The hyperbolicity of knots $K(2 p+3,2 p+1 ; 2 p+2, n)$ for $|n|>3$ follows from the same argument as above, in which we refer to [12, Figure 33] instead of [12, Figure 47].

Proof of Corollary 1.9 Given any torus knot $T_{p, q}(p>q \geq 2)$, let us take an unknotted circle $c=c_{p, q}^{+}$in $S^{3}-T_{p, q}$; see Figure 12(left). Then as shown in the 
proof of Theorem 1.8, an $n$-twist along $c$ converts $T_{p, q}$ into the twisted torus knot $K(p, q ; p+q, n)$, which is an L-space knot for all integers $n$ and hyperbolic if $|n|>3$. The last assertion of the corollary follows from Claim 5.4 below. Thus the unknotted circle $c$ satisfies the required property.

Claim 5.4 $\{K(p, q ; p+q, n)\}_{|n|>3}$ is a set of mutually distinct hyperbolic knots.

Proof Recall that $c_{p, q}^{+}$is a seiferter for $\left(T_{p, q}, p q\right)$ and the linking number between $c_{p, q}^{+}$and $T_{p, q}$ is $p+q$. Thus an $n$-twist along $c_{p, q}^{+}$changes $\left(T_{p, q}, p q\right)$ to a Seifert surgery $\left(K(p, q ; p+q, n), p q+n(p+q)^{2}\right)$. Also, $K(p, q ; p+q, n)\left(p q+n(p+q)^{2}\right)$ is a Seifert fiber space over $S^{2}$ with at most three exceptional fibers of indices $p, q$ and $|n|$, see the proof of Theorem 1.8.

Assume that $K(p, q ; p+q, n)$ is isotopic to $K\left(p, q ; p+q, n^{\prime}\right)$ for some integers $n$ and $n^{\prime}$ with $|n|,\left|n^{\prime}\right|>3$. Then $p q+n(p+q)^{2}-$ and $p q+n^{\prime}(p+q)^{2}$-surgeries on the hyperbolic knot $K(p, q ; p+q, n)$ yield Seifert fiber spaces. Hence,

$$
\left|p q+n(p+q)^{2}-\left(p q+n^{\prime}(p+q)^{2}\right)\right|=\left|\left(n-n^{\prime}\right)(p+q)^{2}\right| \leq 8,
$$

by [32, Theorem 1.2]. Since $p+q \geq 5$, we have $n=n^{\prime}$. This completes the proof. (In the above argument, we can apply [1, Theorem 8.1] which gives the bound 10 instead of 8.)

\section{L-space twisted Berge knots}

In this section we prove Theorem 1.11 using Theorem 1.7 and observations in [13; 12].

Proof of Theorem 1.11 Berge [5] gave twelve infinite families of knots which admit lens space surgeries. These knots are referred to as Berge knots of types (I)-(XII) and are conjectured to comprise all knots with lens space surgeries. Recall that a Berge knot of type (I) is a torus knot and that of (II) is a cable of a torus knot, henceforth we consider Berge knots of types (III)-(XII).

Berge knots of types (III)-(VI) Suppose that $K$ is a Berge knot of type (III), (IV), (V) or (VI). Then we have an unknotted solid torus $V$ containing $K$ in its interior such that $V(K ; m)$ is a solid torus $[5 ; 12]$, and hence the core $c$ of the solid torus $W=S^{3}$-int $V$ is a seiferter for $(K, m)$, and $\left(K_{n}, m_{n}\right)$ is also a lens space. If $K_{n}\left(m_{n}\right)$ is not an L-space, then it is $S^{2} \times S^{1}$, and $\left(K_{n}, m_{n}\right)=(O, 0)$ by [17, Theorem 8.1]. Now let us exclude this possibility. First we note that $V\left(K_{n}, m_{n}\right) \cong V(K ; m)$ for all integers $n$, and $H_{1}\left(V\left(K_{n} ; m_{n}\right)\right) \cong \mathbb{Z} \oplus \mathbb{Z}_{\left(m_{n}, \omega\right)}$ [20, Lemma 3.3], where $\omega$ is 


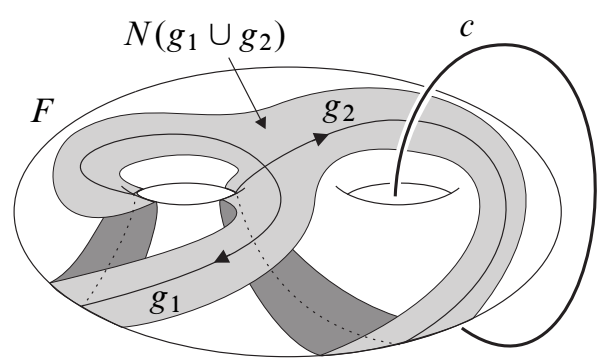

Figure 17: A regular neighborhood $N\left(g_{1} \cup g_{2}\right)$ of $g_{1} \cup g_{2}$ in $F$ and an unknotted circle $c$.

the winding number of $K$ in $V$, ie the linking number between $K_{n}$ and $c$. Since $V\left(K_{n} ; m_{n}\right) \cong S^{1} \times D^{2}, K_{n}$ is a 0 or 1 -bridge braid in $V$ [18]; hence $\omega \geq 2$. This then implies that $m_{n} \neq 0$. Hence $\left(K_{n}, m_{n}\right)$ is an L-space knot for all integers $n$.

Berge knots of types (VII) and (VIII) Let $g_{1}$ and $g_{2}$ be simple closed curves embedded in a genus-two Heegaard surface $F$ of $S^{3}$ and $c$ an unknot in $S^{3}$ as in Figure 17.

Take a regular neighborhood $N\left(g_{1} \cup g_{2}\right)$ of $g_{1} \cup g_{2}$ in $F$, which is a once-punctured torus. Then the curve $\partial N\left(g_{1} \cup g_{2}\right)$ becomes a trefoil knot after a (-1)-twist along $c$, and the figure-eight knot after a 1-twist along $c$. Let $k$ be a knot in $N\left(g_{1} \cup g_{2}\right)$ representing $a\left[g_{1}\right]+b\left[g_{2}\right] \in H_{1}\left(N\left(g_{1} \cup g_{2}\right)\right)$, where $a$ and $b$ are coprime integers. Then we see that $k$ is a torus knot $T_{a+b,-a}$. The Berge knot $K$ of type (VII) (resp. (VIII)) is obtained from $T_{a+b,-a}$ by a (-1)-twist (resp. 1-twist) along $c$. As shown in [13, Lemma 4.6], $T_{a+b,-a} \cup c$ is isotopic to $T_{a+b,-a} \cup c_{a+b,-a}^{+}$, and a Berge knot of type (VII) is $K(a+b,-a ;|b|,-1)$, while that of type (VIII) is $K(a+b,-a ;|b|, 1)$; see the proof of Theorem 1.8. (Here we extend the notation $K(p, q ; r, n)$ for twisted torus knots in an obvious fashion to include the case where $p, q$ are possibly negative integers.)

We assume $|a|,|b| \geq 2$, for otherwise $K(a+b,-a,|b|, \pm 1)$ is a torus knot. Furthermore, if $|a+b|=1$, then $T_{a+b,-a} \cup c=T_{ \pm 1,-a} \cup c$ is a torus link $T_{2,2 b}$ or $T_{2,-2 b}$, and $K(a+b,-a ;|b|, \pm 1)$ is a torus knot, so we assume $|a+b|>1$. Let $K_{n}$ be a knot obtained from the Berge knot $K$ by an $n$-twist along $c$, ie $K_{n}=K(a+b,-a ;|b|, n+\varepsilon)$; $\varepsilon=-1$ if $K$ is of type (VII) and $\varepsilon=1$ if $K$ is of type (VIII). If $a(a+b)<0$ (ie $-a(a+b)>0$ ), then by Theorem 1.8, $K_{n}$ is an L-space knot for any integer $n$. If $a(a+b)>0$ (ie $-a(a+b)<0)$, Theorem 1.8 shows that the mirror image $K(a+b, a ;|b|,-n-\varepsilon)$ of $K_{n}$ is an L-space knot if $-n-\varepsilon \geq-1$, ie $n \leq 1-\varepsilon$. Hence $K_{n}$ is an L-space knot for any integer $n \leq 1-\varepsilon$.

Berge knots of types (IX)-(XII) These knots are often called sporadic knots, and we denote them by Spor $\boldsymbol{a}[p]$, Spor $\boldsymbol{b}[p]$, Spor $\boldsymbol{c}[p]$ and Spor $\boldsymbol{d}[p]$, respectively, where 
$p \geq 0$. It is easy to see that Spor $\boldsymbol{a}[0]$ and Spor $\boldsymbol{b}[0]$ are trivial knots, Spor $\boldsymbol{c}[0]=T_{-3,4}$ and Spor $\boldsymbol{d}[0]=T_{-5,3}$. Thus we may assume $p>0$. Furthermore, we observe that Spor $\boldsymbol{a}[1]$ is obtained from $T_{3,2}$ by a 1 -twist along the seiferter $c=c_{3,2}^{+}$; see Figure 9. Hence, following Example 4.6, a knot $K_{n}$ obtained from Spor $a[1]$ by an $n$-twist along $c$ is an L-space knot for any integer $n$. Thus we may assume $p>1$ for Spor $\boldsymbol{a}[p]$.

As shown in Example 4.7, the lens space surgery (Spor $\boldsymbol{a}[p], 22 p^{2}+9 p+1$ ) is obtained from $\left(C_{6 p+1, p}\left(T_{3,2}\right), p(6 p+1)\right)$ by a 1 -twist along the seiferter $c$, and an $n$-twist along $c$ converts $C_{6 p+1, p}\left(T_{3,2}\right)$ into an L-space knot for all integers $n$. Hence an $n$-twist changes Spor $\boldsymbol{a}[p]$ to an L-space knot for all integers $n$.

The proof of Theorem 1.8 shows that the lens space surgery (Spor $\boldsymbol{b}[p], 22 p^{2}+13 p+2$ ) is obtained from $\left(T_{3 p+1,2 p+1},(3 p+1)(2 p+1)\right)$ by a 1 -twist along the seiferter $c$; hence we obtain $K_{n}=K(3 p+1,2 p+1 ; 4 p+1, n+1)$ by performing an $n$-twist on Spor $\boldsymbol{b}[p]$ along $c$. By Theorem $1.8, K_{n}$ is an L-space knot for all integers $n$. Similarly, (Spor $\left.c[p],-22 p^{2}-31 p-11\right)$ is obtained from $\left(T_{-3 p-2,2 p+1},(-3 p-2)(2 p+1)\right.$ ) by a $(-1)$-twist along $c^{\prime}$, and $K_{n}$, obtained from Spor $c[p]$ by an $n$-twist along $c^{\prime}$, is $K(-3 p-2,2 p+1 ; 4 p+4, n-1)$. Theorem 1.8 shows that its mirror image $K(3 p+2,2 p+1 ; 4 p+4,-n+1)$ is an L-space knot for any integer $n$, and thus $K_{n}$ is an L-space knot for all integers $n$.

Finally, let us consider a Berge knot Spor $\boldsymbol{d}[p](p \geq 0)$. By [12, Proposition 8.8], the lens space surgery (Spor $\boldsymbol{d}[p],-22 p^{2}-35 p-14$ ) has a seiferter $c^{\prime}$ such that the linking number between $c^{\prime}$ and Spor $\boldsymbol{d}[p]$ is $4 p+3$, and a 1 -twist along $c^{\prime}$ converts (Spor $\left.\boldsymbol{d}[p],-22 p^{2}-35 p-14\right)$ into $\left(C_{-6 p-5, p+1}\left(T_{-3,2}\right),(-6 p-5)(p+1)\right)$, for which $c^{\prime}$ is a seiferter. Let $K_{n}$ be a knot obtained from Spor $\boldsymbol{d}[p]$ by an $n$-twist along $c^{\prime}$, ie obtained from $C_{-6 p-5, p+1}\left(T_{-3,2}\right)$ by an $(n-1)$-twist along $c^{\prime}$. Now we take the mirror image of $C_{-6 p-5, p+1}\left(T_{-3,2}\right) \cup c^{\prime}$ to obtain a link $C_{6 p+5, p+1}\left(T_{3,2}\right) \cup c$. Then $c$ is a seiferter for $\left(C_{6 p+5, p+1}\left(T_{3,2}\right),(6 p+5)(p+1)\right)$, and $K_{n}$ is the mirror image of the knot obtained from $C_{6 p+5, p+1}\left(T_{3,2}\right)$ by a $(-n)-$ twist along $c$. Since $(4 p+3)^{2} \geq 2(6 p+5)(p+1)$, Theorem 1.7 shows that $K_{n}$ is an L-space knot for all integers $n$.

Let us show that $K_{n}$ is a hyperbolic knot except for at most four integers $n$. Following [13, Theorem 5.10], it is sufficient to observe that $K \cup c$ is a hyperbolic link. Suppose that $K$ is a Berge knot of type (III), (IV), (V) or (VI). Then as mentioned above, $V(K ; m)$ is a solid torus, where $V=S^{3}-\operatorname{int} N(c)$. By [6, Theorem 3.2], $V-\operatorname{int} N(K)$ is atoroidal. If $V-\operatorname{int} N(K)$ is not hyperbolic, then it is Seifert fibered and $K$ is a torus knot; see [13, Lemma 3.3]. This contradicts the assumption. Hence $K \cup c$ is a hyperbolic link. 

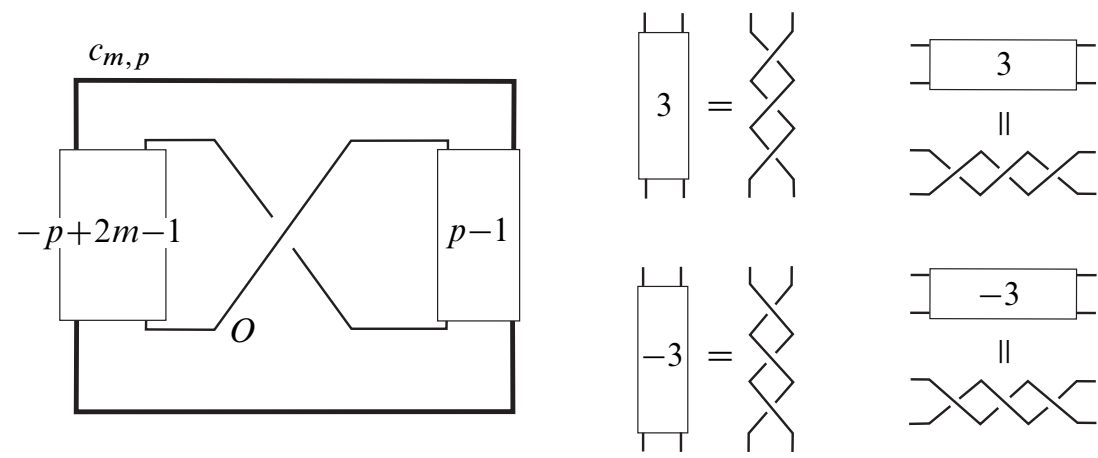

Figure 18: $O \cup c_{m, p}$; a vertical (resp. horizontal) box with integer $n$ denotes a vertical (resp. horizontal) stack of $n$ crossings.

If $K$ is of type (VII) or (VIII), then $K \cup c \cong T_{a+b,-a} \cup c_{a+b,-a}^{+}$is a hyperbolic link; see the proof of Theorem 1.8.

Assume that $K$ is of type (IX), ie $K=$ Spor $a[p]$. Then as shown in the proof of Example 4.7, $K \cup c$ is a hyperbolic link. In the case where $K$ is of type (X) or (XI), ie $K=\operatorname{Spor} \boldsymbol{b}[p]$ or Spor $\boldsymbol{c}[p]$, it follows from the proof of Theorem 1.8 that $K \cup c$ is a hyperbolic link. The argument in the proof of Example 4.7 shows that $K \cup c$ is a hyperbolic link for a type (XII) Berge knot $K=\operatorname{Spor} d[p]$; we refer to [12, Figure 53] instead of [12, Figure 41].

This completes the proof of Theorem 1.11.

\section{L-space twisted unknots}

In [13] we introduced the " $m$-move" to find seiferters for a given Seifert surgery. In particular, the $m$-move is effectively used in [13, Theorem 6.21] to show that $(O, m)$ has infinitely many seiferters for each integer $m$. Among them, there are infinitely many seiferters $c$ such that the $(m, 0)$-surgery on $O \cup c$ is an L-space; see Remark 7.3.

Let us take a trivial knot $c_{m, p}$ in $S^{3}-O$ as illustrated in Figure 18, where $p$ is an odd integer with $|p| \geq 3$.

Then as shown in [13, Theorem 6.21], $c_{m, p}$ is a seiferter for $(O, m)$ such that $O \cup c_{m, p}$ is a hyperbolic link in $S^{3}$ if $p \neq 2 m \pm 1$. Denote by $K_{m, p, n}$ and $m_{p, n}$ the images of $O$ and $m$, respectively, after an $n$-twist along $c_{m, p}$. Now we investigate $K_{m, p, n}\left(m_{p, n}\right)$ using branched coverings and the Montesinos trick [38; 39]. Figure 19(upper-right) shows that $K_{m, p, n}\left(m_{p, n}\right)$ has an involution with axis $L$ for any integer $n$. Taking the quotient by this involution, we obtain a 2 -fold branched cover $\pi: K_{m, p, n}\left(m_{p, n}\right) \rightarrow S^{3}$ 

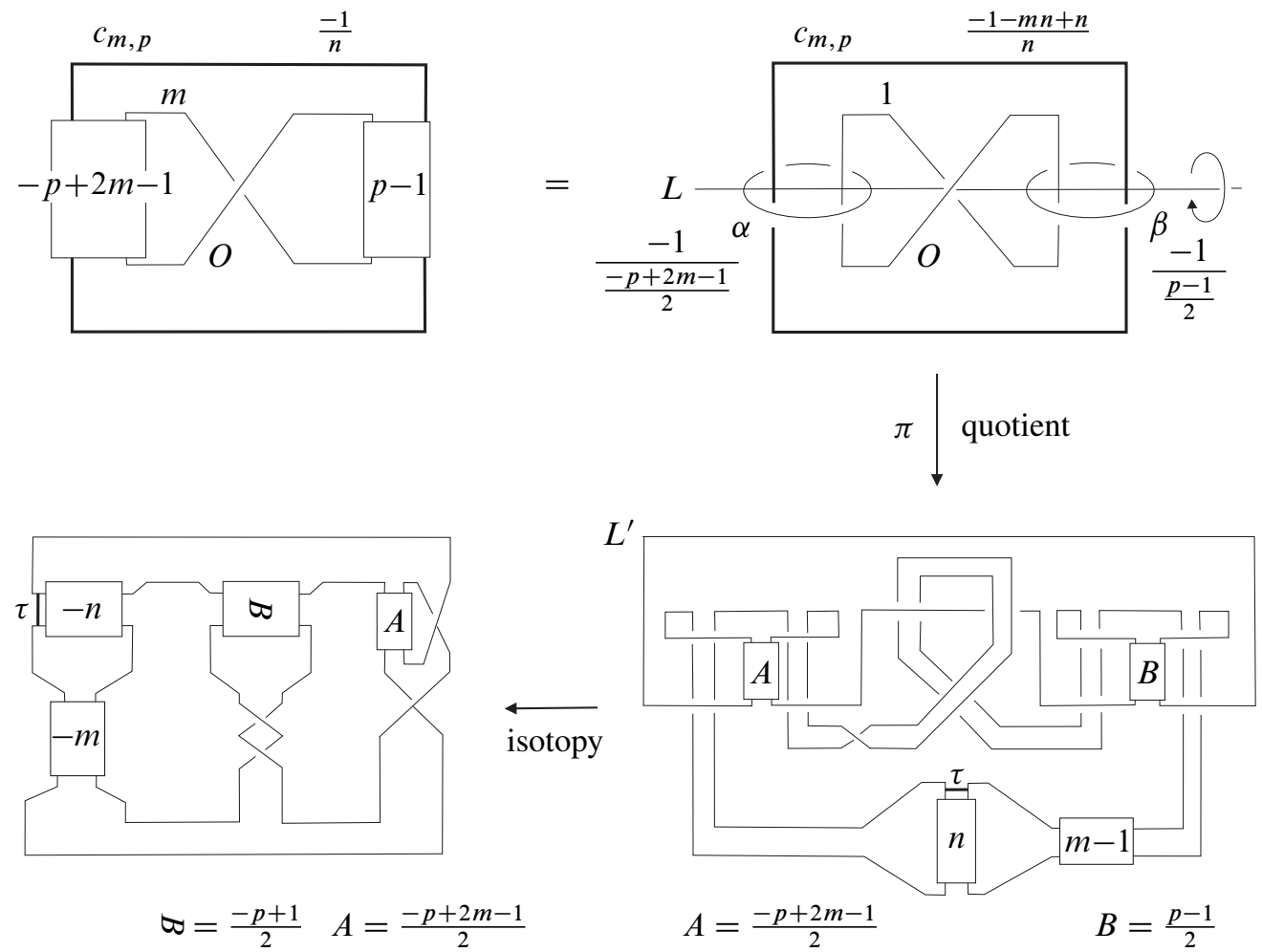

$\varpi=\frac{-p+1}{2} \quad A=\frac{-p+2 m-1}{2}$

$$
A=\frac{-p+2 m-1}{2} \quad B=\frac{p-1}{2}
$$

Figure 19: $K_{m, p, n}\left(m_{p, n}\right)$ is the two-fold branched cover of $S^{3}$ branched along $L^{\prime}$.

branched along $L^{\prime}$, the quotient of $L$; see Figure 19(lower-right). Then $L^{\prime}$ can be isotoped to a Montesinos link

$$
M(-n /(m n+1),(-p+1) / 2 p,(-p+2 m+1) /(-2 p+4 m))
$$

as shown in Figure 19(lower-left). Hence by [38], $K_{m, p, n}\left(m_{p, n}\right)$, which is the 2-fold branched cover branched along the Montesinos link $L^{\prime}$, is a Seifert fiber space

$$
S^{2}\left(\frac{-n}{m n+1}, \frac{-p+1}{2 p}, \frac{p-2 m-1}{2 p-4 m}\right) .
$$

The image $\pi\left(c_{m, p}\right)$ is an arc $\tau$ whose ends lie in $L^{\prime}$; see Figure 19(lower-right) and (lower-left). It follows from [11, Lemma 3.2] that $c_{m, p}$ is a seiferter for $\left(K_{m, p, n}, m_{p, n}\right)$; in case of $n=0, c_{m, p}$ is a seiferter for $(O, m)$. In the following, the image of $c_{m, p}$ after an $n$-twist along itself is also denoted by $c_{m, p}$. 
Proposition 7.1 Assume that $m \leq 0$ and $p \geq 3$.

(1) $\left(K_{m, p, n}, m_{p, n}\right)$ is an L-space surgery except when $(m, n)=(0,0)$. If $(m, n)=$ $(0,0)$, then $\left(K_{m, p, n}, m_{p, n}\right)=(O, 0)$ and $K_{m, p, n}\left(m_{p, n}\right)=O(0) \cong S^{2} \times S^{1}$.

(2) $K_{m, p, n}$ is a nontrivial knot if $n \neq 0$.

(3) $\left\{K_{m, p, n}\right\}|n|>1$ is a set of mutually distinct hyperbolic L-space knots.

Proof We note here that the linking number between $c_{m, p}$ and $O$ is $p-m$.

(1) Assume first that $m=0$. Then $K_{m, p, n}\left(m_{p, n}\right)$ is a lens space

$$
S^{2}(-n,(-p+1) / 2 p,(p-1) / 2 p)=S^{2}(-n-1,(p+1) / 2 p,(p-1) / 2 p),
$$

which is $S^{2} \times S^{1}$ if and only if $n=0$ by Claim 2.5. Hence $K_{m, p, n}\left(m_{p, n}\right)$ is an L-space except when $n=0$.

Next assume $m=-1$. Then

$$
\begin{aligned}
K_{m, p, n}\left(m_{p, n}\right) & =S^{2}(-n /(-n+1),(-p+1) / 2 p,(p+1) /(2 p+4)) \\
& =S^{2}(n /(n-1),(-p+1) / 2 p,(p+1) /(2 p+4)) .
\end{aligned}
$$

If $n=0$ or 2 , then $K_{m, p, n}\left(m_{p, n}\right)$ is a lens space, but it is not $S^{2} \times S^{1}$, because $m_{p, n}=-1+n(m-p)^{2}=-1+n(p+1)^{2} \neq 0$. If $n=1, K_{m, p, n}\left(m_{p, n}\right)$ is a connected sum of two lens spaces, and thus an L-space. Suppose that $n \neq 0,1,2$. In the case where $n<0$, we have $0<n /(n-1)<1$ and

$$
\begin{aligned}
K_{m, p, n}\left(m_{p, n}\right) & =S^{2}(n /(n-1),(-p+1) / 2 p,(p+1) /(2 p+4)) \\
& =S^{2}(-1, n /(n-1),(p+1) / 2 p,(p+1) /(2 p+4)) .
\end{aligned}
$$

Note that

$$
\begin{aligned}
(p+1) / 2 p+(p+1) /(2 p+4) & =1 / 2+1 / 2 p+1 / 2-1 /(2 p+4) \\
& =1+1 / 2 p-1 /(2 p+4) .
\end{aligned}
$$

Since $p \geq 3$, we have $2 p+4>2 p>0$, and hence $1 / 2 p-1 /(2 p+4)>0$. It follows that $(p+1) / 2 p+(p+1) /(2 p+4)=1+1 / 2 p-1 /(2 p+4)>1$. Then Lemma $2.3(2)$ shows that $K_{m, p, n}\left(m_{p, n}\right)$ is an L-space. If $n>2$, then $1<n /(n-1)<2$ and

$$
\begin{aligned}
K_{m, p, n}\left(m_{p, n}\right) & =S^{2}(n /(n-1),(-p+1) / 2 p,(p+1) /(2 p+4)) \\
& =S^{2}(1 /(n-1),(p+1) / 2 p,(p+1) /(2 p+4)) .
\end{aligned}
$$

Since $0<1 /(n-1),(p+1) / 2 p,(p-2 m-1) /(2 p-4 m)<1$, by Theorem $2.1(1)$, $K_{m, p, n}\left(m_{p, n}\right)$ is an L-space. 
Assume that $m=-2$. Then

$$
\begin{aligned}
K_{m, p, n}\left(m_{p, n}\right) & =S^{2}(-n /(-2 n+1),(-p+1) / 2 p,(p+3) /(2 p+8)) \\
& =S^{2}(n /(2 n-1),(-p+1) / 2 p,(p+3) /(2 p+8)) .
\end{aligned}
$$

If $n=0$ or 1 , then $K_{m, p, n}\left(m_{p, n}\right)$ is a lens space, but it is not $S^{2} \times S^{1}$, because $m_{p, n}=-2+n(m-p)^{2}=-2+n(p+2)^{2} \neq 0$. Otherwise, $0<n /(2 n-1)<1$ and

$$
\begin{aligned}
K_{m, p, n}\left(m_{p, n}\right) & =S^{2}(n /(2 n-1),(-p+1) / 2 p,(p+3) /(2 p+8)) \\
& =S^{2}(-1, n /(2 n-1),(p+1) / 2 p,(p+3) /(2 p+8)) .
\end{aligned}
$$

Since

$$
\begin{aligned}
(p+1) / 2 p+(p+3) /(2 p+8) & =1 / 2+1 / 2 p+1 / 2-1 /(2 p+8) \\
& =1+1 / 2 p-1 /(2 p+8) \\
& >1,
\end{aligned}
$$

$K_{m, p, n}\left(m_{p, n}\right)$ is an L-space by Lemma 2.3(2).

Finally, assume that $m \leq-3$. Then

$$
\begin{aligned}
K_{m, p, n}\left(m_{p, n}\right) & =S^{2}(-n /(m n+1),(-p+1) / 2 p,(p-2 m-1) /(2 p-4 m)) \\
& =S^{2}(-1,-n /(m n+1),(p+1) / 2 p,(p-2 m-1) /(2 p-4 m)) .
\end{aligned}
$$

If $n=0$, then $K_{m, p, n}\left(m_{p, n}\right)$ is a lens space, but it is not $S^{2} \times S^{1}$, because $m_{p, n}=$ $m+n(m-p)^{2}=m \leq-3$. Assume $n \neq 0$. Then we have $0<-n /(m n+1)<1$, $0<(p+1) / 2 p<1$ and $0<(p-2 m-1) /(2 p-4 m)=1 / 2-1 /(2 p-4 m)<1$ by the assumptions $p \geq 3$ and $m \leq-3$. Since

$$
\begin{aligned}
(p+1) / 2 p+(p-2 m-1) /(2 p-4 m) & =1 / 2+1 / 2 p+1 / 2-1 /(2 p-4 m) \\
& =1+1 / 2 p-1 /(2 p-4 m) \\
& >1,
\end{aligned}
$$

Lemma 2.3(2) shows that $K_{m, p, n}\left(m_{p, n}\right)$ is an L-space.

(2) Since $m \leq 0$ and $p \geq 3$, we have $p \neq 2 m \pm 1$, and hence $O \cup c_{m, p}$ is a hyperbolic link; see [13, Theorem 6.21]. Then $K_{m, p, n}$ is nontrivial for any $n \neq 0$; see [30;35].

(3) By (1), $K_{m, p, n}$ is an L-space knot. Since $O \cup c_{m, p}$ is a hyperbolic link, the hyperbolicity of $K_{m, p, n}$ for $|n|>1$ follows from [2; 21;37]. Thus $K_{m, p, n}(|n|>1)$ is a hyperbolic L-space knot. Let us choose $c_{m, p}$ and then apply an $n$-twist along $c_{m, p}$ to obtain a knot $K_{m, p, n}$. It remains to show that the $K_{m, p, n}$ are distinct knots. Suppose that $K_{m, p, n}$ and $K_{m, p, n^{\prime}}$ are isotopic for some integers $n$ and $n^{\prime}$ with $|n|,\left|n^{\prime}\right|>1$. Then $\left(m+n(p-m)^{2}\right)$ - and $\left(m+n^{\prime}(p-m)^{2}\right)$-surgeries on $K_{m, p, n}=K_{m, p, n^{\prime}}$ produce 
small Seifert fiber spaces, where $p-m \geq 3$. (Note that $m n+1$ cannot be zero since $|n|>1$.) Since $K_{m, p, n}$ is a hyperbolic knot, Lackenby and Meyerhoff prove in [32, Theorem 1.2] that the distance $\mid m+n(p-m)^{2}-\left(m+n^{\prime}(p-m)^{2} \mid\right.$ between these two nonhyperbolic surgeries is at most 8 . Hence $\left|\left(n-n^{\prime}\right)(p-m)^{2}\right| \leq 8$, which implies $n=n^{\prime}$ because $p-m \geq 3$.

Next we investigate link types of $O \cup c_{m, p}$.

Proposition 7.2 Let $c_{m, p}$ and $c_{m^{\prime}, p^{\prime}}$ be seiferters for $(O, m)$ and $\left(O, m^{\prime}\right)$, respectively. Suppose that $m, m^{\prime} \leq 0$ and $p, p^{\prime} \geq 3$.

(1) If $p-m \neq p^{\prime}-m^{\prime}$, then $O \cup c_{m, p}$ and $O \cup c_{m^{\prime}, p^{\prime}}$ are not isotopic. In particular, if $p \neq p^{\prime}$, then $O \cup c_{m, p}$ and $O \cup c_{m, p^{\prime}}$ are not isotopic.

(2) If $p-m=p^{\prime}-m^{\prime}$, then $O \cup c_{m, p}$ and $O \cup c_{m^{\prime}}, p^{\prime}$ are not isotopic provided that $\left|m-m^{\prime}\right|>3$.

Proof (1) Note that the linking number between $c_{m, p}$ and $O$ is $p-m$. Hence if $O \cup c_{m, p}$ is isotopic to $O \cup c_{m^{\prime}, p^{\prime}}$ as ordered links, then we have $p-m=p^{\prime}-m^{\prime}$.

(2) Since $p \neq 2 m \pm 1$ and $p^{\prime} \neq 2 m^{\prime} \pm 1$, both $O \cup c_{m, p}$ and $O \cup c_{m^{\prime}, p^{\prime}}$ are hyperbolic links [13]. Recall that $c_{m, p}$ is a seiferter for $(O, m)$ and $c_{m^{\prime}, p^{\prime}}$ is a seiferter for $\left(O, m^{\prime}\right)$. Suppose that $O \cup c_{m, p}$ and $O \cup c_{m^{\prime}, p^{\prime}}$ are isotopic. Then $c_{m, p}$ is a seiferter for $\left(O, m^{\prime}\right)$ as well. Let $V$ be the solid torus $S^{3}-$ int $N\left(c_{m, p}\right)$, which contains $O$ in its interior. Note that $m$-surgery of $V$ along $O$ yields a Seifert fiber space over the disk with two exceptional fibers of indices $2 p$ and $2 p-4 m$, and $m^{\prime}$-surgery of $V$ along $O$ yields a Seifert fiber space over the disk with two exceptional fibers of indices $2 p^{\prime}$ and $2 p^{\prime}-4 m^{\prime}$. Since these Seifert fiber spaces contain essential annuli, Gordon and $\mathrm{Wu}$ show in [22, Corollary 1.2] that $\left|m-m^{\prime}\right| \leq 3$.

Proof of Theorem 1.10 This follows from Propositions 7.1 and 7.2.

Remark 7.3 For each seiferter $c_{m, p}(m \leq 0, p \geq 3)$, we can see that $M_{c_{m, p}}(O, m)$ is an L-space. In fact, $M_{c_{m, p}}(O, m)$ is the limit of $K_{m, p, n}\left(m_{p, n}\right)$ when $|n|$ tends to $\infty$ (see Remark 3.2), and

$$
\begin{aligned}
M_{c_{m, p}}(O, m) & =S^{2}(-1 / m,(-p+1) / 2 p,(p-2 m-1) /(2 p-4 m)) \\
& =S^{2}(-1,-1 / m,(p+1) / 2 p,(p-2 m-1) /(2 p-4 m)) .
\end{aligned}
$$

If $m=-1,0$, then $M_{c_{m, p}}(O, m)$ is an L-space by Claim 3.4. If $m<-1$, since $(p+1) / 2 p+(p-2 m-1) /(2 p-4 m)=1+1 / 2 p-1 /(2 p-4 m)>1$, we have that $M_{c_{m, p}}(O, m)$ is an L-space. 


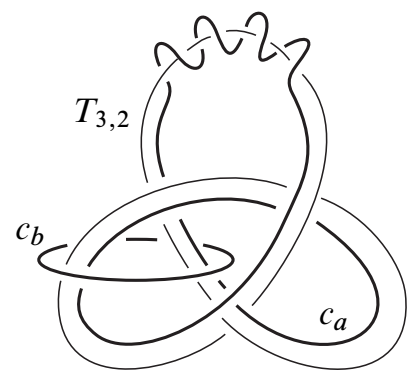

Figure 20: $\left\{c_{a}, c_{b}\right\}$ is a pair of seiferters for $\left(T_{3,2}, 7\right)$.

On the other hand, for instance,

$$
M_{c_{3,3}}(O, 3)=S^{2}(-1 / 3,-1 / 3,2 / 3)=S^{2}(-2,2 / 3,2 / 3,2 / 3),
$$

and taking $k=2$ and $a=1$ in Theorem 2.1(3), we have $(1-2 / 3,1-2 / 3,1-2 / 3)=$ $(1 / 3,1 / 3,1 / 3)<(1 / 2,1 / 2,1 / 2)$. Thus $M_{c_{3,3}}(O, 3)$ is not an L-space.

\section{Hyperbolic L-space knots with tunnel number greater than one}

The purpose in this section is to exhibit infinitely many hyperbolic L-space knots with tunnel number greater than one; see Theorem 1.13. In [16], Eudave-Muñoz, Jasso, Miyazaki and the author gave Seifert fibered surgeries which do not arise from a primitive/Seifert-fibered construction [10].

Let us take unknotted circles $c_{a}$ and $c_{b}$ in $S^{3}-T_{3,2}$ as illustrated by Figure 20. Then as shown in [16], $\left\{c_{a}, c_{b}\right\}$ is a pair of seiferters for $\left(T_{3,2}, 7\right)$, ie $c_{a}$ and $c_{b}$ become fibers simultaneously in some Seifert fibration of $T_{3,2}(7)$.

Note that the pair $\left\{c_{a}, c_{b}\right\}$ forms the $(4,2)$-torus link in $S^{3}$. Hence a $(-1)$-twist along $c_{a}$ converts $c_{a} \cup c_{b}$ into the $(-4,2)$-torus link. Then we can successively apply a 1-twist along $c_{b}$ to obtain the $(4,2)$-torus link $c_{a} \cup c_{b}$. We denote the images of $c_{a}$ and $c_{b}$ under twistings along these components by the same symbols, $c_{a}$ and $c_{b}$, respectively.

Let $K_{n, 0}$ be a knot obtained from $T_{3,2}$ after the sequence of twistings

$$
\left(c_{a},(-1) \text {-twist }\right) \rightarrow\left(c_{b}, 1 \text {-twist }\right) \rightarrow\left(c_{a}, n \text {-twist }\right) .
$$

Then $K_{n, 0}=K(2,-n, 1,0)$ in [16, Proposition 4.11]. See Figure 21.

Similarly, let $K_{0, n}$ be a knot obtained from $T_{3,2}$ after the sequence of twistings

$$
\left(c_{a},(-1) \text {-twist }\right) \rightarrow\left(c_{b},(n+1) \text {-twist }\right) .
$$




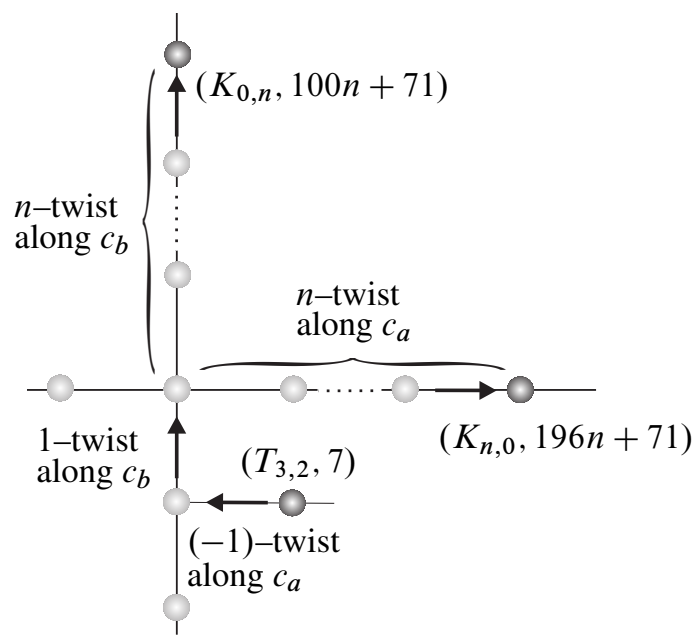

Figure 21: Seifert surgeries $\left(K_{n, 0}, 196 n+71\right)$ and $\left(K_{0, n}, 100 n+71\right)$; each vertex corresponds to a Seifert surgery and each edge corresponds to a single twist along a seiferter.

Then $K_{0, n}=K(2,0,1,-n)$ in [16, Proposition 4.11]. See Figure 21.

Theorem 1.13 follows from Theorem 8.1 below.

Theorem 8.1 (1) $\left\{K_{n, 0}\right\}_{n \in \mathbb{Z}}$ is a set of mutually distinct hyperbolic L-space knots with tunnel number two.

(2) $\left\{K_{0, n}\right\}_{n \in \mathbb{Z} \backslash\{-1\}}$ is a set of mutually distinct hyperbolic L-space knots with tunnel number two.

Proof We begin by recalling the following result, which is a combination of [16, Propositions 3.2, 3.7, 3.11].

Lemma 8.2 (1) $K_{n, 0}$ is a hyperbolic knot with tunnel number two. In addition, $K_{n, 0}(196 n+71)$ is a Seifert fiber space $S^{2}((11 n+4) /(14 n+5),-2 / 7,1 / 2)$.

(2) $K_{0, n}$ is a hyperbolic knot with tunnel number two if $n \neq-1$. In addition, $K_{0, n}(100 n+71)$ is a Seifert fiber space $S^{2}(-(3 n+2) /(10 n+7), 4 / 5,1 / 2)$.

Lemma 8.3 (1) If $K_{n, 0}$ and $K_{n^{\prime}, 0}$ are isotopic, then $n=n^{\prime}$.

(2) If $K_{0, n}$ and $K_{0, n^{\prime}}$ are isotopic, then $n=n^{\prime}$.

Proof of lemma (1) Suppose that $K_{n, 0}$ is isotopic to $K_{n^{\prime}, 0}$. Then $K_{n, 0}(196 n+71)$ and $K_{n, 0}\left(196 n^{\prime}+71\right)$ are both Seifert fiber spaces. Since $K_{n, 0}$ is hyperbolic, we have that $\left|196 n+71-\left(196 n^{\prime}+71\right)\right|=\left|196\left(n-n^{\prime}\right)\right| \leq 8$ from [32, Theorem 1.2]. Hence $n=n^{\prime}$. Part (2) follows in a similar fashion. 
Let us prove that $K_{n, 0}$ and $K_{0, n}$ are L-space knots for any integer $n$.

Lemma 8.4 (1) $K_{n, 0}(196 n+71)$ is an $L$-space for any integer $n$.

(2) $K_{0, n}(100 n+71)$ is an $L$-space for any integer $n$.

Proof of lemma (1) Note that

$$
\begin{aligned}
K_{n, 0}(196 n+71) & =S^{2}((11 n+4) /(14 n+5),-2 / 7,1 / 2) \\
& =S^{2}(-1,(11 n+4) /(14 n+5), 5 / 7,1 / 2) .
\end{aligned}
$$

Since $0<(11 n+4) /(14 n+5)<1$ for any $n \in \mathbb{Z}$ and $5 / 7+1 / 2 \geq 1$, Lemma 2.3(2) shows that $K_{n, 0}(196 n+71)$ is an L-space for any integer $n$. This proves (1).

(2) As above, we first note that

$$
\begin{aligned}
K_{0, n}(100 n+71) & =S^{2}(-(3 n+2) /(10 n+7), 4 / 5,1 / 2) \\
& =S^{2}(-1,(7 n+5) /(10 n+7), 4 / 5,1 / 2) .
\end{aligned}
$$

Since $0<(7 n+5) /(10 n+7)<1$ for any $n \in \mathbb{Z}$ and $4 / 5+1 / 2 \geq 1$, Lemma 2.3(2) shows that $K_{0, n}(100 n+71)$ is an L-space for any integer $n$.

Now Theorem 8.1 follows from Lemmas 8.2, 8.3 and 8.4.

Question 8.5 Does there exist a hyperbolic L-space knot with tunnel number greater than two? More generally, for a given integer $p$, does there exist a hyperbolic L-space knot with tunnel number greater than $p$ ?

\section{Questions}

\section{Characterization of twistings which yield infinitely many L-space knots}

For knots $K$ with Seifert surgery $(K, m)$, Theorems $1.4,1.5,1.6$ and 1.7 characterize seiferters which enjoy the desired property in Question 1.1.

The next proposition, which is essentially shown in $[25 ; 26]$, describes yet another example of twistings which yield infinitely many L-space knots.

Proposition 9.1 (L-space twisted satellite knots) Let $k$ be a nontrivial knot with $L$-space surgery $(k, 2 g-1)$, where $g$ denotes the genus of $k$ and $K$ a satellite knot of $k$ which lies in $V=N(k)$ with winding number $w$. Suppose that $V(K ; m)$ is a solid torus for some integer $m \geq w^{2}(2 g-1)$. Let $c$ be the boundary of a meridian disk of $V$ and $K_{n}$ a knot obtained from $K$ by an $n$-twist along $c$. Then $K_{n}$ is an $L$-space knot for any $n \geq 0$. See Figure 22. 


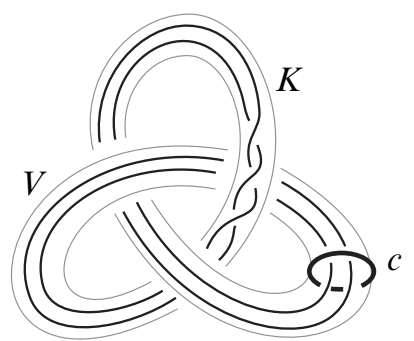

Figure 22: $K_{n}$ is a knot obtained from $K$ by an $n$-twist along $c$.

Proof Recall that $K_{n}\left(m+n w^{2}\right)=k\left(\left(m+n w^{2}\right) / w^{2}\right)=k\left(m / w^{2}+n\right)$ [20]. Since $k(2 g-1)$ is an L-space and $m / w^{2} \geq 2 g-1$, [48, Proposition 9.6] ensures that $k\left(m / w^{2}+n\right)$ is also an L-space if $n \geq 0$. Hence $K_{n}$ is an L-space knot provided $n \geq 0$.

Remark 9.2 (1) In Proposition 9.1, the knot $K$ in the solid torus $V$ is required to have a cosmetic surgery: $V(K ; m) \cong S^{1} \times D^{2}$. The cosmetic surgery of the solid torus is well-understood by $[18 ; 6]$.

(2) The twisting operation described in Proposition 9.1 can be applied only for satellite knots, and the resulting knots after the twistings are also satellite knots.

(3) In Proposition 9.1, the knot $k$ is assumed to be nontrivial. If $k$ is a trivial knot in $S^{3}$, then $K(m)=\left(S^{3}-\right.$ int $\left.V\right) \cup V(K ; m)$ is a lens space; hence $(K, m)$ is an L-space surgery. It is easy to see that $c$ is a seiferter for $(K, m)$.

For further study, we can weaken a condition of seiferter to obtain a notion of "pseudoseiferter" as follows.

Definition 9.3 Let $(K, m)$ be a Seifert surgery. A knot $c$ in $S^{3}-N(K)$ is called a pseudo-seiferter for $(K, m)$ if $c$ satisfies (1) and (2) below.

(1) $c$ is a trivial knot in $S^{3}$.

(2) $c$ becomes a "cable" of a fiber in a Seifert fibration of $K(m)$, and the preferred longitude $\lambda$ of $c$ in $S^{3}$ becomes the cabling slope of $c$ in $K(m)$.

We do not know if a pseudo-seiferter exists, but if $(K, m)$ admits a pseudo-seiferter, it behaves like a seiferter in the following sense. Let $V$ be a fibered tubular neighborhood of a fiber $t$, and let $c$ be a cable in $V$. Then the result of a surgery (corresponding to an $n$-twist) on $c$ of $V$ is again a solid torus, and this surgery is reduced to a surgery on the fiber $t$, which is a core of $V$. Hence $K_{n}\left(m_{n}\right)$ is a (possibly degenerate) Seifert 
fiber space. This suggests that a pseudo-seiferter is also a candidate for an unknotted circle as described in Question 1.1.

We would like to ask the following question for nonsatellite knots.

Question 9.4 Let $K$ be a nonsatellite knot and $K_{n}$ a knot obtained from $K$ by an $n$-twist along an unknotted circle $c$ in $S^{3}-K$. Suppose that the twist family $\left\{K_{n}\right\}$ contains infinitely many L-space knots.

(1) Does $K$ admit a Seifert surgery $(K, m)$ for which $c$ is a seiferter?

(2) Does $K$ admit a Seifert surgery $(K, m)$ for which $c$ is a seiferter or a pseudoseiferter?

\section{L-space knots and strong invertibility}

A knot is said to be strongly invertible if there exists an orientation-preserving involution of $S^{3}$ which fixes the knot setwise and reverses orientation. Known L-space knots are strongly invertible, so it is natural to ask:

Problem 9.5 (Watson) Are L-space knots strongly invertible?

In [13], an "asymmetric seiferter" defined below is essentially used to find Seifert fibered surgery on knots with no symmetry.

Definition 9.6 A seiferter $c$ for a Seifert surgery $(K, m)$ is said to be symmetric if we have an orientation preserving diffeomorphism $f: S^{3} \rightarrow S^{3}$ of finite order with $f(K)=K$ and $f(c)=c$; otherwise, $c$ is called an asymmetric seiferter.

Combining [13, Theorem 7.3] and Theorem 1.4, we obtain:

Proposition 9.7 Let $(K, m)$ be a Seifert fibered surgery on a nonsatellite knot with an asymmetric seiferter $c$ which becomes an exceptional fiber. Suppose that $M_{c}(K, m)$ is an $L$-space. Then there is a constant $N$ such that $K_{n}$, a knot obtained from $K$ by an $n$-twist along $c$, is a hyperbolic $L$-space knot either with no symmetry for any $n \leq N$ or with no symmetry for any $n \geq N$.

If $c$ is a seiferter for $\left(T_{p, q}, p q\right)$ which becomes a degenerate fiber in $T_{p, q}(p q)$, then $c$ is a meridian of $T_{p, q}$ or $T_{p, q} \cup c$ is a hyperbolic link in $S^{3}$; see [13, Theorem 3.19 (3)]. Hence the argument in the proof of [13, Theorem 7.3] and Theorem 1.6(2) enable us to show: 
Proposition 9.8 If $c$ is an asymmetric seiferter for $\left(T_{p, q}, p q\right)$ which becomes a degenerate fiber in $T_{p, q}(p q)$, then there is a constant $N$ such that $K_{n}$ is a hyperbolic $L$-space knot either with no symmetry for any $n \leq N$ or with no symmetry for any $n \geq N$.

For the asymmetric seiferter $c=c_{1}^{\prime}$ for $(K, m)=(P(-3,3,5), 1)$ given in [13, Lemma 7.5], $M_{c}(K, m)$ is not an L-space, and $c$ does not satisfy the hypothesis of Proposition 9.7.

Question 9.9 Does there exist an asymmetric seiferter as described in Propositions 9.7 and 9.8 ?

\section{References}

[1] I Agol, Bounds on exceptional Dehn filling, Geom. Topol. 4 (2000) 431-449 MR

[2] M Aït-Nouh, D Matignon, K Motegi, Geometric types of twisted knots, Ann. Math. Blaise Pascal 13 (2006) 31-85 MR

[3] K Baker, A Moore, Montesinos knots, Hopf plumbings, and L-space surgeries, preprint (2014) arXiv

[4] R Benedetti, C Petronio, Lectures on hyperbolic geometry, Springer, Berlin (1992) MR

[5] J Berge, Some knots with surgeries yielding lens spaces, unpublished manuscript

[6] J Berge, The knots in $D^{2} \times S^{1}$ which have nontrivial Dehn surgeries that yield $D^{2} \times S^{1}$, Topology Appl. 38 (1991) 1-19 MR

[7] M Boileau, J Porti, Geometrization of 3-orbifolds of cyclic type, Astérisque 272, Société Mathématique de France, Paris (2001) MR

[8] S Boyer, C M Gordon, L Watson, On L-spaces and left-orderable fundamental groups, Math. Ann. 356 (2013) 1213-1245 MR

[9] S Boyer, D Rolfsen, B Wiest, Orderable 3-manifold groups, Ann. Inst. Fourier (Grenoble) 55 (2005) 243-288 MR

[10] J C Dean, Small Seifert-fibered Dehn surgery on hyperbolic knots, Algebr. Geom. Topol. 3 (2003) 435-472 MR

[11] A Deruelle, M Eudave-Muñoz, K Miyazaki, K Motegi, Networking Seifert surgeries on knots, IV: Seiferters and branched coverings, from: "Geometry and topology down under", (C D Hodgson, W H Jaco, M G Scharlemann, S Tillmann, editors), Contemp. Math. 597, Amer. Math. Soc. (2013) 235-262 MR

[12] A Deruelle, K Miyazaki, K Motegi, Networking Seifert surgeries on knots, II: The Berge's lens surgeries, Topology Appl. 156 (2009) 1083-1113 MR 
[13] A Deruelle, K Miyazaki, K Motegi, Networking Seifert surgeries on knots, Mem. Amer. Math. Soc. 1021, Providence, RI (2012) MR

[14] D Eisenbud, U Hirsch, W Neumann, Transverse foliations of Seifert bundles and self-homeomorphism of the circle, Comment. Math. Helv. 56 (1981) 638-660 MR

[15] M Eudave-Muñoz, On hyperbolic knots with Seifert fibered Dehn surgeries, from: "Proceedings of the First Joint Japan-Mexico Meeting in Topology", (S García-Ferreira, T Nogura, editors), volume 121 (2002) 119-141 MR

[16] M Eudave-Muñoz, E Jasso, K Miyazaki, K Motegi, Seifert fibered surgeries on strongly invertible knots without primitive/Seifert positions, Topology Appl. 196 (2015) 729-753 MR

[17] D Gabai, Foliations and the topology of 3-manifolds, III, J. Differential Geom. 26 (1987) 479-536 MR

[18] D Gabai, Surgery on knots in solid tori, Topology 28 (1989) 1-6 MR

[19] P Ghiggini, Knot Floer homology detects genus-one fibred knots, Amer. J. Math. 130 (2008) 1151-1169 MR

[20] C M Gordon, Dehn surgery and satellite knots, Trans. Amer. Math. Soc. 275 (1983) 687-708 MR

[21] C M Gordon, J Luecke, Non-integral toroidal Dehn surgeries, Comm. Anal. Geom. 12 (2004) 417-485 MR

[22] CM Gordon, Y-Q Wu, Annular Dehn fillings, Comment. Math. Helv. 75 (2000) 430-456 MR

[23] J E Greene, L-space surgeries, genus bounds, and the cabling conjecture, J. Differential Geom. 100 (2015) 491-506 MR

[24] A Hatcher, Notes on basic 3-manifold topology, unfinished book manuscript, Cornell University (2007) Available at http://www.math.cornell.edu/ hatcher/3M/ $3 \mathrm{Mfds} \cdot \mathrm{pdf}$

[25] M Hedden, On knot Floer homology and cabling, II, Int. Math. Res. Not. 2009 (2009) 2248-2274 MR

[26] J Hom, T Lidman, F Vafaee, Berge-Gabai knots and L-space satellite operations, Algebr. Geom. Topol. 14 (2014) 3745-3763 MR

[27] W Jaco, Lectures on three-manifold topology, CBMS Regional Conference Series in Mathematics 43, Amer. Math. Soc. (1980) MR

[28] M Jankins, W D Neumann, Rotation numbers of products of circle homeomorphisms, Math. Ann. 271 (1985) 381-400 MR

[29] A Juhász, Floer homology and surface decompositions, Geom. Topol. 12 (2008) 299_ $350 \mathrm{MR}$ 
[30] M Kouno, K Motegi, T Shibuya, Twisting and knot types, J. Math. Soc. Japan 44 (1992) 199-216 MR

[31] D T Krcatovich, The reduced knot Floer complex, PhD thesis, Michigan State University (2014) MR Available at http://search.proquest.com/docview/ 1561147250

[32] M Lackenby, R Meyerhoff, The maximal number of exceptional Dehn surgeries, Invent. Math. 191 (2013) 341-382 MR

[33] T Lidman, A H Moore, Pretzel knots with L-space surgeries, Michigan Math. J. 65 (2016) 105-130 MR

[34] P Lisca, A I Stipsicz, Ozsváth-Szabó invariants and tight contact 3-manifolds, III, J. Symplectic Geom. 5 (2007) 357-384 MR

[35] Y Mathieu, Unknotting, knotting by twists on disks and property (P) for knots in $S^{3}$, from: "Knots 90”, (A Kawauchi, editor), de Gruyter, Berlin (1992) 93-102 MR

[36] K Miyazaki, K Motegi, Seifert fibred manifolds and Dehn surgery, Topology 36 (1997) 579-603 MR

[37] K Miyazaki, K Motegi, Seifert fibered manifolds and Dehn surgery, III, Comm. Anal. Geom. 7 (1999) 551-582 MR

[38] J M Montesinos, Seifert manifolds that are ramified two-sheeted cyclic coverings, Bol. Soc. Mat. Mexicana 18 (1973) 1-32 MR

[39] J M Montesinos, Surgery on links and double branched covers of $S^{3}$, from: "Knots, groups, and 3-manifolds (Papers dedicated to the memory of R H Fox)", (L P Neuwirth, editor), Ann. of Math. Studies 84, Princeton Univ. Press (1975) 227-259 MR

[40] K Morimoto, M Sakuma, On unknotting tunnels for knots, Math. Ann. 289 (1991) 143-167 MR

[41] K Motegi, K Tohki, On L-space twisted torus knots, in preparation

[42] R Naimi, Foliations transverse to fibers of Seifert manifolds, Comment. Math. Helv. 69 (1994) 155-162 MR

[43] Y Ni, Knot Floer homology detects fibred knots, Invent. Math. 170 (2007) 577-608 MR

[44] Y Ni, Erratum: Knot Floer homology detects fibred knots, Invent. Math. 177 (2009) 235-238 MR

[45] P Ozsváth, Z Szabó, Holomorphic disks and genus bounds, Geom. Topol. 8 (2004) 311-334 MR

[46] P Ozsváth, Z Szabó, Holomorphic disks and three-manifold invariants: properties and applications, Ann. of Math. 159 (2004) 1159-1245 MR

[47] P Ozsváth, Z Szabó, On knot Floer homology and lens space surgeries, Topology 44 (2005) 1281-1300 MR 
[48] PS Ozsváth, Z Szabó, Knot Floer homology and rational surgeries, Algebr. Geom. Topol. 11 (2011) 1-68 MR

[49] C Petronio, J Porti, Negatively oriented ideal triangulations and a proof of Thurston's hyperbolic Dehn filling theorem, Expo. Math. 18 (2000) 1-35 MR

[50] Z Szabó, Lecture notes on Heegaard Floer homology, from: "Low dimensional topology”, (T S Mrowka, P S Ozsváth, editors), IAS/Park City Math. Ser. 15, Amer. Math. Soc. (2009) 197-228 MR

[51] W P Thurston, The geometry and topology of three-manifolds, lecture notes, Princeton University (1979) Available at http://library.msri.org/books/gt3m/

[52] W P Thurston, Three-dimensional manifolds, Kleinian groups and hyperbolic geometry, Bull. Amer. Math. Soc. 6 (1982) 357-381 MR

[53] F Vafaee, On the knot Floer homology of twisted torus knots, Int. Math. Res. Not. 2015 (2015) 6516-6537 MR

Department of Mathematics, Nihon University

3-25-40 Sakurajosui, Setagaya-ku, Tokyo 156-8550, Japan

motegi@math.chs.nihon-u.ac.jp

http://www.math.chs.nihon-u.ac.jp/ motegi/

Received: 28 April $2015 \quad$ Revised: 16 August 2015 